\title{
Entropy numbers of embeddings of function spaces with Muckenhoupt weights, III. Some limiting cases
}

\section{Dorothee D. Haroske and Leszek Skrzypczak}

(Communicated by Hans Triebel)

2000 Mathematics Subject Classification. 46E35.

Keywords and phrases. Besov spaces, Triebel-Lizorkin spaces, Muckenhoupt weights, compact embeddings, entropy numbers.

Abstract. We study compact embeddings for weighted spaces of Besov and Triebel-Lizorkin type where the weight belongs to some Muckenhoupt $\mathcal{A}_{p}$ class. This extends our previous results [25] to more general weights of logarithmically disturbed polynomial growth, both near some singular point and at infinity. We obtain sharp asymptotic estimates for the entropy numbers of this embedding. Essential tools are a discretisation in terms of wavelet bases, as well as a refined study of associated embeddings in sequence spaces and interpolation arguments in endpoint situations.

\section{Introduction}

In recent years, some attention has been paid to compactness of embeddings of function spaces of Besov and Sobolev type as well as to analytic and geometric quantities describing this compactness, in particular, corresponding approximation and entropy numbers. In [13] Edmunds and Triebel proposed a program to investigate the 
spectral properties of certain pseudo-differential operators based on the asymptotic behaviour of entropy and approximation numbers, together with Carl's inequality and the Birman-Schwinger principle. Similar questions in the context of weighted function spaces of this type were studied by the first named author and Triebel, cf. [19], and were continued and extended by Kühn, Leopold, Sickel and the second author in the series of papers [31-33]. All these papers were devoted to the class of so-called 'admissible' weights: These are smooth weights with no singular points, with $w(x)=\left(1+|x|^{2}\right)^{\alpha / 2}$, $\alpha \in \mathbb{R}, x \in \mathbb{R}^{n}$, as a prominent example.

We started in [25] a different approach and considered weights from the Muckenhoupt class $\mathcal{A}_{\infty}$ which - unlike 'admissible' weights - may have local singularities, that can influence embedding properties of such function spaces. Weighted Besov and Triebel-Lizorkin spaces with Muckenhoupt weights are well known concepts, cf. [4-7], [15,39,40] and, more recently, [2,3], [24]. But (the compactness of) their embeddings were not yet studied in detail.

In [25] we dealt with general transformation methods from function to appropriate sequence spaces provided by a wavelet decomposition; we essentially concentrated on the example weight

$$
w_{\alpha, \beta}(x) \sim\left\{\begin{array}{ll}
|x|^{\alpha} & \text { if }|x| \leq 1, \\
|x|^{\beta} & \text { if } \quad|x|>1,
\end{array} \quad \text { with } \quad \alpha>-n, \quad \beta>0,\right.
$$

of purely polynomial growth both near the origin and for $|x| \rightarrow \infty$. In the general setting for $w \in \mathcal{A}_{\infty}$ we obtained sharp criteria for the continuity or compactness of embeddings of type

$$
\text { id }: A_{p_{1}, q_{1}}^{s_{1}}\left(\mathbb{R}^{n}, w\right) \hookrightarrow A_{p_{2}, q_{2}}^{s_{2}}\left(\mathbb{R}^{n}\right),
$$

where $s_{2} \leq s_{1}, 0<p_{1}, p_{2}<\infty, 0<q_{1}, q_{2} \leq \infty$, and $A_{p, q}^{s}$ stands for either Besov spaces $B_{p, q}^{s}$ or Triebel-Lizorkin spaces $F_{p, q}^{s}$. Moreover, for $w=w_{\alpha, \beta}$ given by (1.1) we determined the exact asymptotic behaviour of corresponding entropy and approximation numbers, e.g.,

$e_{k}\left(\operatorname{id}: A_{p_{1}, q_{1}}^{s_{1}}\left(\mathbb{R}^{n}, w_{\alpha, \beta}\right) \hookrightarrow A_{p_{2}, q_{2}}^{s_{2}}\left(\mathbb{R}^{n}\right)\right) \sim k^{-\min \left(\frac{\beta}{n p_{1}}+\frac{1}{p_{1}}-\frac{1}{p_{2}}, \frac{s_{1}-s_{2}}{n}\right)}, \quad k \in \mathbb{N}$, if $\frac{\beta}{n p_{1}}+\frac{1}{p_{1}}-\frac{1}{p_{2}} \neq \frac{s_{1}-s_{2}}{n}$. (There are further results in [25] also dealing with 'double-weighted' situations and corresponding approximation numbers.) It turned out that the 'local' singularity (represented 
by the parameter $\alpha$ here) has no further influence on the 'degree' of compactness (measured in terms of entropy or approximation numbers, respectively). We investigated this phenomenon in [26] in detail, introducing the new concept of the set of singularities $\mathbf{S}_{\text {sing }}(w)$ which - roughly speaking - collects all those $x_{0} \in \mathbb{R}^{n}$ where the weight tends to infinity or vanishes. We could prove in [26] that the entropy and approximation numbers (for spaces on bounded domains) do not take care of this singular behaviour of the weight as long as $\mathbf{S}_{\mathrm{sing}}(w)$ is bounded and 'small' enough.

In both above contributions [25,26] we were directly led to questions of limiting embeddings which could not be solved in the respective context. This is the reason for us to return to this subject in further detail, before we come to applications in the above sense (but out of the scope of the present paper).

Instead of the weight $w_{\alpha, \beta}$ given by (1.1) we now consider its refinement

$$
w_{(\boldsymbol{\alpha}, \boldsymbol{\beta})}(x)=\left\{\begin{array}{lll}
|x|^{\alpha_{1}}(1-\log |x|)^{\alpha_{2}}, & \text { if } & |x| \leq 1, \\
|x|^{\beta_{1}}(1+\log |x|)^{\beta_{2}}, & \text { if } & |x|>1,
\end{array}\right.
$$

where

(1.3) $\boldsymbol{\alpha}=\left(\alpha_{1}, \alpha_{2}\right), \alpha_{1}>-n, \alpha_{2} \in \mathbb{R}, \quad \boldsymbol{\beta}=\left(\beta_{1}, \beta_{2}\right), \beta_{1}>-n, \beta_{2} \in \mathbb{R}$.

Our first main result in Proposition 3.9 is the complete characterisation of the continuity and compactness of

$$
\text { id }: B_{p_{1}, q_{1}}^{s_{1}}\left(\mathbb{R}^{n}, w_{(\boldsymbol{\alpha}, \boldsymbol{\beta})}\right) \hookrightarrow B_{p_{2}, q_{2}}^{s_{2}}\left(\mathbb{R}^{n}\right) .
$$

It is compact if, and only if,

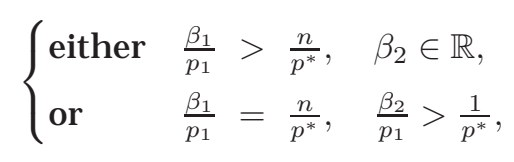

and

$$
\begin{cases}\text { either } & \delta>\max \left(\frac{\alpha_{1}}{p_{1}}, \frac{n}{p^{*}}\right), \quad \alpha_{2} \in \mathbb{R} \\ \text { or } & \delta=\frac{\alpha_{1}}{p_{1}}>\frac{n}{p^{*}}, \quad \frac{\alpha_{2}}{p_{1}}>\frac{1}{q^{*}}\end{cases}
$$

where $\frac{1}{p^{*}}=\max \left(\frac{1}{p_{2}}-\frac{1}{p_{1}}, 0\right), \frac{1}{q^{*}}=\max \left(\frac{1}{q_{2}}-\frac{1}{q_{1}}, 0\right)$, and $\delta=s_{1}-$ $\frac{n}{p_{1}}-s_{2}+\frac{n}{p_{2}}$. Since $w_{(\boldsymbol{\alpha}, \boldsymbol{\beta})}$ with $\alpha_{2}=\beta_{2}=0$ coincides with $w_{\alpha_{1}, \beta_{1}}$ 
in the sense of (1.1) and studied in [25], it is clear that the limiting cases $\frac{\beta_{1}}{p_{1}}=\frac{n}{p^{*}}$ in (1.5) and $\delta=\frac{\alpha_{1}}{p_{1}}$ in (1.6) were excluded so far. It is especially interesting (and new in this context) to observe the interplay of the fine indices $q_{1}, q_{2}$ with the local fine parameter $\alpha_{2}$ of the weight $w_{(\boldsymbol{\alpha}, \boldsymbol{\beta})}$ in (1.6). Such phenomena for Besov spaces were already known from similar considerations. We essentially concentrate on one-weighted situations here, but refer to doubleweighted counterparts occasionally.

Some new idea is connected with Besov or Triebel-Lizorkin spaces built upon Lorentz-Zygmund spaces $L_{p, r}(\log L)_{a}\left(\mathbb{R}^{n}\right)$, thus extending the usual approach based on $L_{p}\left(\mathbb{R}^{n}\right)=L_{p, p}(\log L)_{0}\left(\mathbb{R}^{n}\right)$. These spaces appeared in special settings in [12] already and to some extent - in [8,13] (for bounded domains) and [18,21] (on $\mathbb{R}^{n}$ ). Another more abstract approach can be found in [10]. In the present paper these spaces $A_{q}^{s}\left(L_{p, r}(\log L)_{a}\right)\left(\mathbb{R}^{n}\right)$ appear naturally as appropriate endpoint spaces for our weight $w_{(\boldsymbol{\alpha}, \boldsymbol{\beta})}$. We shall only sketch some ideas now and apply particular results, but we have the strong feeling that these spaces deserve a more systematic and detailed study, both from the abstract interpolation and extrapolation point of view, and for applications.

Our second main result can be found in Proposition 4.4 where we study entropy numbers of the embeddings of certain sequence spaces,

$$
\operatorname{id}^{\beta_{1}, \beta_{2}}: \ell_{q_{1}}\left(2^{j \delta} \ell_{p_{1}}\left(w^{\beta_{1}, \beta_{2}}\right)\right) \hookrightarrow \ell_{q_{2}}\left(\ell_{p_{2}}\right)
$$

with

$$
w^{\beta_{1}, \beta_{2}}(j, m)=\left(1+2^{-j}|m|\right)^{\beta_{1}}\left(1+\log \left(1+2^{-j}|m|\right)\right)^{\beta_{2}}, \quad j \in \mathbb{N}_{0}, m \in \mathbb{Z}^{n},
$$

for $\beta_{1} \geq 0, \beta_{2} \in \mathbb{R}$. This obviously corresponds to embeddings of weighted function spaces not regarding local singularities. We achieve an almost complete description of the asymptotic behaviour of the entropy numbers $e_{k}\left(\mathrm{id}^{\beta_{1}, \beta_{2}}\right)$ for $k \rightarrow \infty$, which is partially based on earlier results in [30-33] and [29], but also covers new cases. These estimates together with local observations are combined to characterise

$$
e_{k}\left(\mathrm{id}: A_{p_{1}, q_{1}}^{s_{1}}\left(\mathbb{R}^{n}, w_{(\boldsymbol{\alpha}, \boldsymbol{\beta})}\right) \hookrightarrow A_{p_{2}, q_{2}}^{s_{2}}\left(\mathbb{R}^{n}\right)\right)
$$

for $k \in \mathbb{N}$; this is presented in Theorem 4.7. In particular, we do not only extend the weight class considered in [25] to logarithmic 
perturbations, but can now deal with the limiting situations $\frac{\beta_{1}}{p_{1}}=\frac{n}{p^{*}}$ in (1.5) and $\delta=\frac{\alpha_{1}}{p_{1}}$ in (1.6) for the first time.

The paper is organised as follows. In Section 2 we recall basic facts about Muckenhoupt weight classes and weighted function spaces needed later on. Section 3 is devoted to the continuity and compactness of the embeddings, in particular, when dealing with $w=w_{(\boldsymbol{\alpha}, \boldsymbol{\beta})}$ given by (1.2) with (1.3). The concluding Section 4 contains our results on the asymptotic behaviour of the entropy numbers for weighted embeddings.

\section{Weighted function spaces}

First of all we need to fix some notation. By $\mathbb{N}$ we denote the set of natural numbers, by $\mathbb{N}_{0}$ the set $\mathbb{N} \cup\{0\}$, and by $\mathbb{Z}^{n}$ the set of all lattice points in $\mathbb{R}^{n}$ having integer components.

The positive part of a real function $f$ is given by $f_{+}(x)=$ $\max (f(x), 0)$. For two positive real sequences $\left\{a_{k}\right\}_{k \in \mathbb{N}}$ and $\left\{b_{k}\right\}_{k \in \mathbb{N}}$ we mean by $a_{k} \sim b_{k}$ that there exist constants $c_{1}, c_{2}>0$ such that $c_{1} a_{k} \leq b_{k} \leq c_{2} a_{k}$ for all $k \in \mathbb{N}$; similarly for positive functions.

Given two (quasi-) Banach spaces $X$ and $Y$, we write $X \hookrightarrow Y$ if $X \subset Y$ and the natural embedding of $X$ in $Y$ is continuous.

All unimportant positive constants will be denoted by $c$, occasionally with subscripts. For convenience, let both $\mathrm{d} x$ and $|\cdot|$ stand for the (n-dimensional) Lebesgue measure in the sequel. If not otherwise indicated, $\log$ is always taken with respect to base 2 .

2.1 Muckenhoupt weights. We briefly recall some fundamentals on Muckenhoupt classes $\mathcal{A}_{p}$. By a weight $w$ we shall always mean a locally integrable function $w \in L_{1}^{\text {loc }}\left(\mathbb{R}^{n}\right)$, positive a.e. in the sequel.

Definition 2.1. Let $w$ be a weight function on $\mathbb{R}^{n}$ and $1<p<\infty$. Then $w$ belongs to the Muckenhoupt class $\mathcal{A}_{p}$, if there exists a constant $0<A<\infty$ such that for all balls $B$ the following inequality holds

$$
\left(\frac{1}{|B|} \int_{B} w(x) \mathrm{d} x\right)^{1 / p}\left(\frac{1}{|B|} \int_{B} w(x)^{-p^{\prime} / p} \mathrm{~d} x\right)^{1 / p^{\prime}} \leq A
$$

where $p^{\prime}$ is the dual exponent to $p$ given by $1 / p^{\prime}+1 / p=1$ and $|B|$ stands for the Lebesgue measure of the ball $B$. 
The limiting cases $p=1$ and $p=\infty$ can be incorporated as follows. Let $M$ stand for the Hardy-Littlewood maximal operator given by

$$
M f(x)=\sup _{B(x, r) \in \mathcal{B}} \frac{1}{|B(x, r)|} \int_{B(x, r)}|f(y)| \mathrm{d} y, \quad x \in \mathbb{R}^{n},
$$

where $\mathcal{B}$ is the collection of all open balls

$$
B(x, r)=\left\{y \in \mathbb{R}^{n}:|y-x|<r\right\}, \quad r>0 .
$$

Definition 2.2. A weight $w$ belongs to the Muckenhoupt class $\mathcal{A}_{1}$ if there exists a constant $0<A<\infty$ such that the inequality

$$
M w(x) \leq A w(x)
$$

holds for almost all $x \in \mathbb{R}^{n}$. The Muckenhoupt class $\mathcal{A}_{\infty}$ is given by

$$
\mathcal{A}_{\infty}=\bigcup_{p>1} \mathcal{A}_{p}
$$

Since the pioneering work of Muckenhoupt [35-37], these classes of weight functions have been studied in great detail, we refer, in particular, to the monographs [16], [44], [45, Ch. IX], and [43, Ch. V] for a complete account on the theory of Muckenhoupt weights. Let us only mention the important feature of decomposition of $\mathcal{A}_{p}$ weights into $\mathcal{A}_{1}$ weights based on the facts that for two $\mathcal{A}_{1}$ weights $w_{1}, w_{2}$, and $1 \leq p<\infty$, then $w=w_{1} w_{2}^{1-p} \in \mathcal{A}_{p}$. Conversely, suppose that $w \in \mathcal{A}_{p}$, then there exist $v_{1} \in \mathcal{A}_{1}, v_{2} \in \mathcal{A}_{1}$ such that $w=v_{1} v_{2}^{1-p}$. Moreover, it is known that the minimum, maximum, and the sum of finitely many $\mathcal{A}_{1}$ weights yields again an $\mathcal{A}_{1}$ weight. We refer to the above-mentioned literature for proofs and further details. As usual, we use the abbreviation

$$
w(\Omega)=\int_{\Omega} w(x) \mathrm{d} x
$$

where $\Omega \subset \mathbb{R}^{n}$ is some bounded, measurable set.

Example 2.3. We restrict ourselves to one typical example only since the study of further weight functions is postponed to the next sections. Let

(2.5) $\quad w(x)=|x|^{\varrho} \log ^{-\varkappa}(2+|x|) \quad$ or $\quad w(x)=|x|^{\varrho} \log ^{\varkappa}\left(2+|x|^{-1}\right)$. 
Then,

$$
w \in \mathcal{A}_{1} \quad \text { if } \quad \begin{cases}\varkappa \in \mathbb{R}, & \text { if }-n<\varrho<0 \\ \varkappa \geq 0, & \text { if } \varrho=0\end{cases}
$$

whereas the counterpart for $1<p<\infty$ reads as

$$
w \in \mathcal{A}_{p} \quad \text { if } \quad-n<\varrho<n(p-1), \quad \varkappa \in \mathbb{R},
$$

see also [14, Lemma 2.3]. In particular,

$$
w_{\varrho}(x)=|x|^{\varrho} \in \mathcal{A}_{p} \quad \text { if } \quad \begin{cases}-n<\varrho \leq 0, & \text { if } p=1, \\ -n<\varrho<n(p-1), & \text { if } 1<p<\infty .\end{cases}
$$

2.2 Function spaces of type $B_{p, q}^{s}\left(\mathbb{R}^{n}, w\right)$ and $F_{p, q}^{s}\left(\mathbb{R}^{n}, w\right)$ with $w \in \mathcal{A}_{\infty}$. Let $w \in \mathcal{A}_{\infty}$ be a Muckenhoupt weight, and $0<$ $p<\infty$. Then the weighted Lebesgue space $L_{p}\left(\mathbb{R}^{n}, w\right)$ contains all measurable functions such that

$$
\left\|f \mid L_{p}\left(\mathbb{R}^{n}, w\right)\right\|=\left(\int_{\mathbb{R}^{n}}|f(x)|^{p} w(x) \mathrm{d} x\right)^{1 / p}
$$

is finite. Note that for $p=\infty$ one obtains the classical (unweighted) Lebesgue space,

$$
L_{\infty}\left(\mathbb{R}^{n}, w\right)=L_{\infty}\left(\mathbb{R}^{n}\right), \quad w \in \mathcal{A}_{\infty} .
$$

Thus we mainly restrict ourselves to $p<\infty$ in what follows.

The Schwartz space $\mathcal{S}\left(\mathbb{R}^{n}\right)$ and its dual $\mathcal{S}^{\prime}\left(\mathbb{R}^{n}\right)$ of all complexvalued tempered distributions have their usual meaning here. Let $\varphi_{0}=\varphi \in \mathcal{S}\left(\mathbb{R}^{n}\right)$ be such that

$$
\operatorname{supp} \varphi \subset\left\{y \in \mathbb{R}^{n}:|y|<2\right\} \quad \text { and } \quad \varphi(x)=1 \quad \text { if } \quad|x| \leq 1,
$$

and for each $j \in \mathbb{N}$ let $\varphi_{j}(x)=\varphi\left(2^{-j} x\right)-\varphi\left(2^{-j+1} x\right)$. Then $\left\{\varphi_{j}\right\}_{j=0}^{\infty}$ forms a smooth dyadic resolution of unity. Given any $f \in \mathcal{S}^{\prime}\left(\mathbb{R}^{n}\right)$, we denote by $\mathcal{F} f$ and $\mathcal{F}^{-1} f$ its Fourier transform and its inverse Fourier transform, respectively. Let $f \in \mathcal{S}^{\prime}\left(\mathbb{R}^{n}\right)$, then the compact support of $\varphi_{j} \mathcal{F} f$ implies by the Paley-Wiener-Schwartz theorem that $\mathcal{F}^{-1}\left(\varphi_{j} \mathcal{F} f\right)$ is an entire analytic function on $\mathbb{R}^{n}$. 
Definition 2.4. Let $0<q \leq \infty, 0<p<\infty, \quad s \in \mathbb{R}$ and $\left\{\varphi_{j}\right\}_{j}$ a smooth dyadic resolution of unity. Assume $w \in \mathcal{A}_{\infty}$.

(i) The weighted Besov space $B_{p, q}^{s}\left(\mathbb{R}^{n}, w\right)$ is the set of all distributions $f \in \mathcal{S}^{\prime}\left(\mathbb{R}^{n}\right)$ such that

$$
\left\|f\left|B_{p, q}^{s}\left(\mathbb{R}^{n}, w\right)\|=\|\left\{2^{j s}\left\|\mathcal{F}^{-1}\left(\varphi_{j} \mathcal{F} f\right) \mid L_{p}\left(\mathbb{R}^{n}, w\right)\right\|\right\}_{j \in \mathbb{N}_{0}}\right| \ell_{q}\right\|
$$

is finite.

(ii) The weighted Triebel - Lizorkin space $F_{p, q}^{s}\left(\mathbb{R}^{n}, w\right)$ is the set of all distributions $f \in \mathcal{S}^{\prime}\left(\mathbb{R}^{n}\right)$ such that

$$
\left\|f \left|F _ { p , q } ^ { s } ( \mathbb { R } ^ { n } , w ) \| = \| \left\|\{ 2 ^ { j s } | \mathcal { F } ^ { - 1 } ( \varphi _ { j } \mathcal { F } f ) ( \cdot ) | \} _ { j \in \mathbb { N } _ { 0 } } \left|\ell_{q}\left\|\mid L_{p}\left(\mathbb{R}^{n}, w\right)\right\|\right.\right.\right.\right.
$$

is finite.

Remark 2.5. The spaces $B_{p, q}^{s}\left(\mathbb{R}^{n}, w\right)$ and $F_{p, q}^{s}\left(\mathbb{R}^{n}, w\right)$ are independent of the particular choice of the smooth dyadic resolution of unity $\left\{\varphi_{j}\right\}_{j}$ appearing in their definitions. They are quasi-Banach spaces (Banach spaces for $p, q \geq 1$ ), and $\mathcal{S}\left(\mathbb{R}^{n}\right) \hookrightarrow$ $B_{p, q}^{s}\left(\mathbb{R}^{n}, w\right) \hookrightarrow \mathcal{S}^{\prime}\left(\mathbb{R}^{n}\right)$, similarly for the $F$-case, where the first embedding is dense if $q<\infty$; cf. [4]. Moreover, for $w_{0} \equiv 1 \in \mathcal{A}_{\infty}$ we obtain the usual (unweighted) Besov and Triebel-Lizorkin spaces; we refer, in particular, to the series of monographs by TRIEBEL [46-49] for a comprehensive treatment of the unweighted spaces.

The above spaces with weights of type $w \in \mathcal{A}_{\infty}$ have been studied systematically by Bui first in [4,5], with subsequent papers [6,7]. It turned out that many of the results from the unweighted situation have weighted counterparts: e.g., we have $F_{p, 2}^{0}\left(\mathbb{R}^{n}, w\right)=h_{p}\left(\mathbb{R}^{n}, w\right)$, $0<p<\infty$, where the latter are Hardy spaces, see [4, Thm. 1.4], and, in particular, $h_{p}\left(\mathbb{R}^{n}, w\right)=L_{p}\left(\mathbb{R}^{n}, w\right)=F_{p, 2}^{0}\left(\mathbb{R}^{n}, w\right), \quad 1<p<\infty$, $w \in \mathcal{A}_{p}$, see [44, Ch. VI, Thm. 1]. Concerning (classical) Sobolev spaces $W_{p}^{k}\left(\mathbb{R}^{n}, w\right)$ built upon $L_{p}\left(\mathbb{R}^{n}, w\right)$ in the usual way, it holds

$$
W_{p}^{k}\left(\mathbb{R}^{n}, w\right)=F_{p, 2}^{k}\left(\mathbb{R}^{n}, w\right), \quad k \in \mathbb{N}_{0}, \quad 1<p<\infty, \quad w \in \mathcal{A}_{p},
$$

cf. [4, Thm. 2.8]. Further results can be found in $[4,5,16,40]$. In [41] the above class of weights was extended to the class $\mathcal{A}_{p}^{\text {loc }}$. Recent works are due to Roudenko [15, 39, 40] and Bownik [2,3]. We partly rely on our approaches [24-26].

We shall need an extension of the (unweighted) spaces $A_{p, q}^{s}\left(\mathbb{R}^{n}\right)$ to some logarithmic spaces $A_{q}^{s}\left(L_{p, r}(\log L)_{a}\right)\left(\mathbb{R}^{n}\right)$ built upon LorentzZygmund spaces. 
By this we mean the modification of (2.12) and (2.13) (with $w \equiv 1$ ) when $L_{p}\left(\mathbb{R}^{n}\right)$ is replaced by the Lorentz-Zygmund space $L_{p, r}(\log L)_{a}\left(\mathbb{R}^{n}\right), 0<p<\infty, 0<r \leq \infty, a \in \mathbb{R}$, given by

$$
\left\|f \mid L_{p, r}(\log L)_{a}\left(\mathbb{R}^{n}\right)\right\| \sim\left(\int_{0}^{\infty}\left[t^{1 / p}(1+|\log t|)^{a} f^{*}(t)\right]^{r} \frac{\mathrm{d} t}{t}\right)^{1 / r}
$$

(appropriately modified if $r=\infty$ ), where $f^{*}$ is the non-increasing rearrangement of $f$, as usual,

$$
f^{*}(t)=\inf \left\{s \geq 0:\left|\left\{x \in \mathbb{R}^{n}:|f(x)|>s\right\}\right| \leq t\right\}, \quad t \geq 0 .
$$

Plainly, $L_{p, r}(\log L)_{0}\left(\mathbb{R}^{n}\right)=L_{p, r}\left(\mathbb{R}^{n}\right)$ with the special case $L_{p, p}(\log L)_{0}\left(\mathbb{R}^{n}\right)=$ $L_{p}\left(\mathbb{R}^{n}\right)$.

Definition 2.6. Let $0<q \leq \infty, \quad 0<p<\infty, \quad s \in \mathbb{R}, 0<r \leq \infty$, $a \in \mathbb{R}$, and $\left\{\varphi_{j}\right\}_{j}$ a smooth dyadic resolution of unity.

(i) The logarithmic Besov space $B_{q}^{s}\left(L_{p, r}(\log L)_{a}\right)\left(\mathbb{R}^{n}\right)$ is the set of all distributions $f \in \mathcal{S}^{\prime}\left(\mathbb{R}^{n}\right)$ such that

$$
\left\|f\left|B_{q}^{s}\left(L_{p, r}(\log L)_{a}\right)\left(\mathbb{R}^{n}\right)\|=\|\left\{2^{j s}\left\|\mathcal{F}^{-1}\left(\varphi_{j} \mathcal{F} f\right) \mid L_{p, r}(\log L)_{a}\left(\mathbb{R}^{n}\right)\right\|\right\}_{j \in \mathbb{N}_{0}}\right| \ell_{q}\right\|
$$

is finite.

(ii) The logarithmic Triebel-Lizorkin space $F_{q}^{s}\left(L_{p, r}(\log L)_{a}\right)\left(\mathbb{R}^{n}\right)$ is the set of all distributions $f \in \mathcal{S}^{\prime}\left(\mathbb{R}^{n}\right)$ such that

$$
\left\|f\left|F_{q}^{s}\left(L_{p, r}(\log L)_{a}\right)\left(\mathbb{R}^{n}\right)\|=\|\left\{2^{j s}\left\|\mathcal{F}^{-1}\left(\varphi_{j} \mathcal{F} f\right)(\cdot) \mid \ell_{q}\right\|\right\}_{j \in \mathbb{N}_{0}}\right| L_{p, r}(\log L)_{a}\left(\mathbb{R}^{n}\right)\right\|
$$

is finite.

Remark 2.7. The above spaces appear in their $F$-version in case of $p=r$ already in [12, Def. 4.3], denoted there by $F_{p, q}^{s}(\log F)_{a}\left(\mathbb{R}^{n}\right)$. In case of $p=r$ and $q=2$ these are the logarithmic Sobolev spaces considered also in $[8,13]$ (for bounded domains) and $[18,21]$ (on $\mathbb{R}^{n}$ ). Their $B$-counterparts were discussed in some personal communication with $\mathrm{H}$. Triebel; see also the general abstract result [10]. However, we shall reserve the notation $A_{p, q}^{s}(\log A)_{a}\left(\mathbb{R}^{n}\right)$ for the spaces obtained by extrapolation and (logarithmic) interpolation according to [10]. Since their coincidence (even on bounded domains and with additional assumptions) is not yet proved in full generality we stick to the clumsy notation introduced above. 
Finally, we briefly describe the wavelet characterisations of Besov spaces with $\mathcal{A}_{\infty}$ weights proved in [25]. Let for $m \in \mathbb{Z}^{n}$ and $\nu \in \mathbb{N}_{0}$, $Q_{\nu, m}$ denote the $n$-dimensional cube with sides parallel to the axes of coordinates, centered at $2^{-\nu} m$ and with side length $2^{-\nu}$. Apart from function spaces with weights we introduce sequence spaces with weights. For $0<p<\infty, 0<q \leq \infty, \sigma \in \mathbb{R}$, and $w \in \mathcal{A}_{\infty}$, we introduce suitable sequence spaces

$$
\begin{aligned}
b_{p, q}^{\sigma}(w):= & \left\{\lambda=\left\{\lambda_{\nu, m}\right\}_{\nu, m}: \lambda_{\nu, m} \in \mathbb{C},\right. \\
& \left.\left\|\lambda\left|b_{p, q}^{\sigma}(w)\|\sim\|\left\{2^{\nu \sigma}\left(\sum_{m \in \mathbb{Z}^{n}}\left|\lambda_{\nu, m}\right|^{p} 2^{\nu n} w\left(Q_{\nu, m}\right)\right)^{\frac{1}{p}}\right\}_{\nu \in \mathbb{N}_{0}}\right| \ell_{q}\right\|<\infty\right\}
\end{aligned}
$$

and

$$
\ell_{p}(w):=\left\{\lambda=\left\{\lambda_{m}\right\}: \lambda_{m} \in \mathbb{C},\left\|\lambda \mid \ell_{p}(w)\right\| \sim\left(\sum_{m \in \mathbb{Z}^{n}}\left|\lambda_{m}\right|^{p} 2^{\nu n} w\left(Q_{0, m}\right)\right)^{\frac{1}{p}}<\infty\right\} .
$$

If $w \equiv 1$ we write $b_{p, q}^{\sigma}$ instead of $b_{p, q}^{\sigma}(w)$.

Let $\tilde{\phi} \in C^{N_{1}}(\mathbb{R})$ be an orthogonal scaling function on $\mathbb{R}$ with $\operatorname{supp} \widetilde{\phi} \subset\left[-N_{2}, N_{2}\right]$ for certain natural numbers $N_{1}$ and $N_{2}$, and $\widetilde{\psi}$ an associated wavelet. Then the tensor-product ansatz yields a scaling function $\phi$ and associated wavelets $\psi_{1}, \ldots, \psi_{2^{n}-1}$, all defined now on $\mathbb{R}^{n}$. This implies

$\phi, \psi_{i} \in C^{N_{1}}\left(\mathbb{R}^{n}\right) \quad$ and $\quad \operatorname{supp} \phi, \operatorname{supp} \psi_{i} \subset\left[-N_{3}, N_{3}\right]^{n}, \quad i=1, \ldots, 2^{n}-1$.

Using the standard abbreviations $\phi_{\nu, m}(x)=2^{\nu n / 2} \phi\left(2^{\nu} x-m\right)$ and $\psi_{i, \nu, m}(x)=2^{\nu n / 2} \psi_{i}\left(2^{\nu} x-m\right)$ we proved in [25, Thm. 1.13] the following wavelet decomposition result.

Theorem 2.8. Let $0<p, q \leq \infty$ and let $s \in \mathbb{R}$. Let $\phi$ be a scaling function and let $\psi_{i}, i=1, \ldots, 2^{n}-1$, be the corresponding wavelets satisfying (2.18). We assume that $|s|<N_{1}$. Then a distribution $f \in \mathcal{S}^{\prime}\left(\mathbb{R}^{n}\right)$ belongs to $B_{p, q}^{s}\left(\mathbb{R}^{n}, w\right)$, if, and only if,

$$
\begin{aligned}
\left\|f \mid B_{p, q}^{s}\left(\mathbb{R}^{n}, w\right)\right\|^{\star}= & \left\|\left\{\left\langle f, \phi_{0, m}\right\rangle\right\}_{m \in \mathbb{Z}^{n}} \mid \ell_{p}(w)\right\| \\
& +\sum_{i=1}^{2^{n}-1}\left\|\left\{\left\langle f, \psi_{i, \nu, m}\right\rangle\right\}_{\nu \in \mathbb{N}_{0}, m \in \mathbb{Z}^{n}} \mid b_{p, q}^{\sigma}(w)\right\|
\end{aligned}
$$


is finite, where $\sigma=s+\frac{n}{2}-\frac{n}{p}$. Furthermore, $\left\|f \mid B_{p, q}^{s}\left(\mathbb{R}^{n}, w\right)\right\|^{\star}$ may be used as an equivalent (quasi-) norm in $B_{p, q}^{s}\left(\mathbb{R}^{n}, w\right)$.

\section{Continuity and compactness of embeddings}

We collect some embedding results for weighted spaces of the above type that will be used later. For that purpose we adopt the nowadays usual custom to write $A_{p, q}^{s}$ instead of $B_{p, q}^{s}$ or $F_{p, q}^{s}$, respectively, when both scales of spaces are meant simultaneously in some context (but always with the understanding of the same choice within one and the same embedding, if not otherwise stated explicitly).

Proposition 3.1. Let $0<q \leq \infty, 0<p<\infty, \quad s \in \mathbb{R}$ and $w \in \mathcal{A}_{\infty}$.

(i) Let $-\infty<s_{1} \leq s_{0}<\infty$ and $0<q_{0} \leq q_{1} \leq \infty$, then

$$
A_{p, q}^{s_{0}}\left(\mathbb{R}^{n}, w\right) \hookrightarrow A_{p, q}^{s_{1}}\left(\mathbb{R}^{n}, w\right), \quad \text { and } \quad A_{p, q_{0}}^{s}\left(\mathbb{R}^{n}, w\right) \hookrightarrow A_{p, q_{1}}^{s}\left(\mathbb{R}^{n}, w\right) .
$$

(ii) We have

$$
B_{p, \min (p, q)}^{s}\left(\mathbb{R}^{n}, w\right) \hookrightarrow F_{p, q}^{s}\left(\mathbb{R}^{n}, w\right) \hookrightarrow B_{p, \max (p, q)}^{s}\left(\mathbb{R}^{n}, w\right) .
$$

(iii) Assume that there are numbers $c>0, d>0$ such that for all balls,

$$
w(B(x, r)) \geq c r^{d}, \quad 0<r \leq 1, \quad x \in \mathbb{R}^{n} .
$$

Let $0<p_{0}<p<p_{1}<\infty,-\infty<s_{1}<s<s_{0}<\infty$ satisfy

$$
s_{0}-\frac{d}{p_{0}}=s-\frac{d}{p}=s_{1}-\frac{d}{p_{1}} \text {. }
$$

Then

$$
\begin{aligned}
B_{p_{0}, q}^{s_{0}}\left(\mathbb{R}^{n}, w\right) & \hookrightarrow B_{p_{1}, q}^{s_{1}}\left(\mathbb{R}^{n}, w\right), \\
F_{p_{0}, \infty}^{s_{0}, \infty}\left(\mathbb{R}^{n}, w\right) & \hookrightarrow F_{p_{1}, q}^{s_{1}}\left(\mathbb{R}^{n}, w\right),
\end{aligned}
$$

and

$$
B_{p_{0}, p}^{s_{0}}\left(\mathbb{R}^{n}, w\right) \hookrightarrow F_{p, q}^{s}\left(\mathbb{R}^{n}, w\right) \hookrightarrow B_{p_{1}, p}^{s_{1}}\left(\mathbb{R}^{n}, w\right) .
$$

Remark 3.2. These embeddings are natural extensions from the unweighted case $w \equiv 1$, see [46, Prop. 2.3.2/2, Thm. 2.7.1] 
and [42, Thm. 3.2.1]. The above result essentially coincides with [4, Thm. 2.6] and can be found in [25, Prop. 1.8]. We shall benefit from this result inasmuch as we can essentially concentrate on Besov spaces which are usually easier to handle, see also Theorem 2.8. This does not apply to limiting cases as will be pointed out later.

We next recall a general result on weighted embeddings and apply it to our model case afterwards.

Proposition 3.3. Let $w_{1}$ and $w_{2}$ be two $\mathcal{A}_{\infty}$ weights and let $-\infty<s_{2} \leq s_{1}<\infty, 0<p_{1}, p_{2} \leq \infty, 0<q_{1}, q_{2} \leq \infty$. We put

$$
\frac{1}{p^{*}}:=\left(\frac{1}{p_{2}}-\frac{1}{p_{1}}\right)_{+} \quad \text { and } \quad \frac{1}{q^{*}}:=\left(\frac{1}{q_{2}}-\frac{1}{q_{1}}\right)_{+} .
$$

(i) There is a continuous embedding $B_{p_{1}, q_{1}}^{s_{1}}\left(\mathbb{R}^{n}, w_{1}\right) \hookrightarrow B_{p_{2}, q_{2}}^{s_{2}}\left(\mathbb{R}^{n}, w_{2}\right)$ if, and only if,

$$
\left\{2^{-\nu\left(s_{1}-s_{2}\right)}\left\|\left\{\left(w_{2}\left(Q_{\nu, m}\right)\right)^{1 / p_{2}}\left(w_{1}\left(Q_{\nu, m}\right)\right)^{-1 / p_{1}}\right\}_{m \in \mathbb{Z}^{n}} \mid \ell_{p *}\right\|\right\}_{\nu \in \mathbb{N}_{0}} \in \ell_{q^{*}} .
$$

(ii) The embedding $B_{p_{1}, q_{1}}^{s_{1}}\left(\mathbb{R}^{n}, w_{1}\right) \hookrightarrow B_{p_{2}, q_{2}}^{s_{2}}\left(\mathbb{R}^{n}, w_{2}\right)$ is compact if, and only if, (3.9) holds and, in addition,

$$
\begin{aligned}
& \lim _{\nu \rightarrow \infty} 2^{-\nu\left(s_{1}-s_{2}\right)}\left\|\left\{\left(w_{2}\left(Q_{\nu, m}\right)\right)^{1 / p_{2}}\left(w_{1}\left(Q_{\nu, m}\right)\right)^{-1 / p_{1}}\right\}_{m \in \mathbb{Z}^{n}} \mid \ell_{p *}\right\|=0 \quad \text { if } q^{*}=\infty, \\
& \text { and } \\
& \lim _{|m| \rightarrow \infty}\left(w_{2}\left(Q_{\nu, m}\right)\right)^{1 / p_{2}}\left(w_{1}\left(Q_{\nu, m}\right)\right)^{-1 / p_{1}}=0 \quad \text { for all } \nu \in \mathbb{N}_{0} \quad \text { if } p^{*}=\infty .
\end{aligned}
$$

Remark 3.4. The result is proved in [25, Prop. 2.1] based on the wavelet decomposition Theorem 2.8, the commutative diagram

$$
\begin{array}{ccc}
B_{p_{1}, q_{1}}^{s_{1}}\left(\mathbb{R}^{n}, w_{1}\right) & \underset{T^{-1}}{\stackrel{T}{\rightleftharpoons}} & b_{p_{1}, q_{1}}^{\sigma_{1}}\left(w_{1}\right) \\
\text { Id } \downarrow & & \downarrow \text { id } \\
B_{p_{2}, q_{2}}^{s_{2}}\left(\mathbb{R}^{n}, w_{2}\right) & \stackrel{S}{\stackrel{S-1}{S^{-1}}} & b_{p_{2}, q_{2}}^{\sigma_{2}}\left(w_{2}\right)
\end{array}
$$

with appropriate isomorphisms $S$ and $T$ and the general result [32, Theorem 1]. Similarly, with an appropriate isomorphism $A$ one can reduce the investigation of the embeddings of two weighted sequence spaces to the study of embeddings of a weighted space 
into an unweighted one, using

$$
\begin{array}{ccc}
b_{p_{1}, q_{1}}^{\sigma_{1}}\left(w_{1}\right) & \stackrel{A}{\rightleftharpoons} & b_{p_{1}, q_{1}}^{\sigma_{1}}\left(w_{1} / w_{2}\right) \\
\text { Id } & & \downarrow \text { id } \\
b_{p_{2}, q_{2}}^{\sigma_{2}}\left(w_{2}\right) & \stackrel{A^{-1}}{\leftrightarrows} & b_{p_{2}, q_{2}}^{\sigma_{2}}
\end{array}
$$

Remark 3.5. In view of (2.10) it is clear that we obtain unweighted Besov spaces if $p_{1}=p_{2}=\infty$. Then by (2.4), $w_{1}\left(Q_{\nu, m}\right)=$ $w_{2}\left(Q_{\nu, m}\right)=2^{-\nu n}$ for all $\nu \in \mathbb{N}_{0}$ and $m \in \mathbb{Z}^{n}$, such that (3.9) leads to $p^{*}=\infty$, i.e., $p_{1} \leq p_{2}$, and

$$
\delta:=s_{1}-\frac{n}{p_{1}}-s_{2}+\frac{n}{p_{2}}>0,
$$

with the extension to $\delta=0$ if $q_{1} \leq q_{2}$, i.e., $q^{*}=\infty$. Moreover, by (3.11), the embedding is never compact (as is well-known in this case).

Thus we may restrict ourselves to the situation when only the source space is weighted, and the target space unweighted,

$$
B_{p_{1}, q_{1}}^{s_{1}}\left(\mathbb{R}^{n}, w\right) \hookrightarrow B_{p_{2}, q_{2}}^{s_{2}}\left(\mathbb{R}^{n}\right),
$$

where $w \in \mathcal{A}_{\infty}$. Moreover, we shall assume in the sequel that $p_{1}<$ $\infty$ for convenience, as otherwise we have $B_{p_{1}, q_{1}}^{s_{1}}\left(\mathbb{R}^{n}, w\right)=B_{p_{1}, q_{1}}^{s_{1}}\left(\mathbb{R}^{n}\right)$, recall (2.10), and we arrive at the unweighted situation in (3.13) which is well-known already. Therefore we stick to the general assumptions

$$
-\infty<s_{2} \leq s_{1}<\infty, \quad 0<p_{1}<\infty, \quad 0<p_{2} \leq \infty, \quad 0<q_{1}, q_{2} \leq \infty .
$$

For later use we recall the corresponding result for a weight of type

$$
w_{\alpha_{1}, \beta_{1}}(x)= \begin{cases}|x|^{\alpha_{1}}, & \text { if }|x| \leq 1, \\ |x|^{\beta_{1}}, & \text { if }|x|>1,\end{cases}
$$

with $\alpha_{1}>-n, \beta_{1}>-n$, where we already adapted the notation appropriately for later comparison with our model weight function. Obviously this modifies Example 2.3. In [25, Prop. 2.6] we proved the following.

Proposition 3.6. Let $w_{\alpha_{1}}, w_{\beta_{1}}$ be given by (2.8), respectively, and $w_{\alpha_{1}, \beta_{1}}$ by (3.15). 
(i) Let $w_{\alpha_{1}} \in \mathcal{A}_{r}$ and $w_{\beta_{1}} \in \mathcal{A}_{r}, 1 \leq r<\infty$, then $w_{\alpha_{1}, \beta_{1}} \in \mathcal{A}_{r}$.

(ii) Let the parameters be given by (3.14). The embedding $B_{p_{1}, q_{1}}^{s_{1}}\left(\mathbb{R}^{n}, w_{\alpha_{1}, \beta_{1}}\right) \hookrightarrow B_{p_{2}, q_{2}}^{s_{2}}\left(\mathbb{R}^{n}\right)$ is continuous if, and only if,

$$
\begin{cases}\text { either } & \beta_{1} \geq 0 \text { if } p^{*}=\infty, \\ \text { or } & \frac{\beta_{1}}{p_{1}}>\frac{n}{p^{*}} \text { if } p^{*}<\infty,\end{cases}
$$

and one of the following conditions is satisfied,

$$
\begin{aligned}
& \delta \geq \max \left(\frac{\alpha_{1}}{p_{1}}, 0\right) \quad \text { if } \quad q^{*}=\infty, p^{*}=\infty, \\
& \delta \geq \max \left(\frac{\alpha_{1}}{p_{1}}, \frac{n}{p^{*}}\right) \quad \text { if } \quad q^{*}=\infty, p^{*}<\infty, \frac{n}{p^{*}} \neq \frac{\alpha_{1}}{p_{1}}, \\
& \delta>\max \left(\frac{\alpha_{1}}{p_{1}}, \frac{n}{p^{*}}\right) \quad \text { otherwise. }
\end{aligned}
$$

(iii) The embedding $A_{p_{1}, q_{1}}^{s_{1}}\left(\mathbb{R}^{n}, w_{\alpha_{1}, \beta_{1}}\right) \hookrightarrow A_{p_{2}, q_{2}}^{s_{2}}\left(\mathbb{R}^{n}\right)$ is compact if, and only if,

$$
\frac{\beta_{1}}{p_{1}}>\frac{n}{p^{*}} \quad \text { and } \quad \delta>\max \left(\frac{n}{p^{*}}, \frac{\alpha_{1}}{p_{1}}\right) .
$$

Remark 3.7. In [25, Prop. 2.8] we have also dealt with the double-weighted situation $B_{p_{1}, q_{1}}^{s_{1}}\left(\mathbb{R}^{n}, w_{\alpha_{1}, \beta_{1}}\right) \hookrightarrow B_{p_{2}, q_{2}}^{s_{2}}\left(\mathbb{R}^{n}, w_{\gamma_{1}, \varkappa_{1}}\right)$. Note that the limiting cases related to the weight, that is, $\frac{\beta_{1}}{p_{1}}=\frac{n}{p^{*}}$ and $\delta=\frac{\alpha_{1}}{p_{1}}$, are excluded in (3.20).

Now we concentrate on our model weight, the following refined version of (3.15) in the spirit of Example 2.3,

$$
w_{(\boldsymbol{\alpha}, \boldsymbol{\beta})}(x):=\left\{\begin{array}{lll}
|x|^{\alpha_{1}}(1-\log |x|)^{\alpha_{2}}, & \text { if } & |x| \leq 1 \\
|x|^{\beta_{1}}(1+\log |x|)^{\beta_{2}}, & \text { if } & |x|>1
\end{array}\right.
$$

where

(3.22) $\boldsymbol{\alpha}=\left(\alpha_{1}, \alpha_{2}\right), \alpha_{1}>-n, \alpha_{2} \in \mathbb{R}, \quad \boldsymbol{\beta}=\left(\beta_{1}, \beta_{2}\right), \beta_{1}>-n, \beta_{2} \in \mathbb{R}$.

We begin with some auxiliary lemma.

Lemma 3.8. Let $\gamma \in \mathbb{R}, \varkappa \in \mathbb{R}, \nu \in \mathbb{N}$. 
(i) If $\gamma>0$, then

$$
\sum_{k=1}^{\nu} 2^{k \gamma} k^{\varkappa} \sim 2^{\nu \gamma} \nu^{\varkappa}
$$

(with equivalence constants independent of $\nu$ ).

(ii) If $\gamma<0$, then

$$
\sum_{k=1}^{\nu} 2^{k \gamma} k^{\varkappa} \sim 1
$$

(with equivalence constants independent of $\nu$ ).

(iii) If $\gamma=0$, then

$$
\sum_{k=1}^{\nu} k^{\varkappa} \sim \begin{cases}1 & , \quad \varkappa<-1 \\ \nu^{1+\varkappa} & , \quad \varkappa>-1 \\ \log (1+\nu) & , \quad \varkappa=-1\end{cases}
$$

(with equivalence constants independent of $\nu$ ).

Proof. The proof is straightforward using only the observation that for arbitrary $\varepsilon>0$ there are constants $c_{1}=c_{1}(\varepsilon, \varkappa)$ and $c_{2}=c_{2}(\varepsilon, \varkappa)$ (but independent of $k$ and $\nu$ ) such that $c_{1}^{-1} \geq 2^{-k \varepsilon} k^{\varkappa} \geq$ $c_{1} 2^{-\nu \varepsilon} \nu^{\varkappa}$, and $c_{2}^{-1} \leq 2^{k \varepsilon} k^{\varkappa} \leq c_{2} 2^{\nu \varepsilon} \nu^{\varkappa}, k=1, \ldots, \nu$.

Proposition 3.9. Let $w_{(\boldsymbol{\alpha}, \boldsymbol{\beta})}$ be given by (3.21), (3.22).

(i) Let $1<r<\infty$ be such that $\max \left(\alpha_{1}, \beta_{1}\right)<n(r-1)$. Then $w_{(\boldsymbol{\alpha}, \boldsymbol{\beta})}$ belongs to $\mathcal{A}_{r}$. Moreover, $w_{(\boldsymbol{\alpha}, \boldsymbol{\beta})} \in \mathcal{A}_{1}$ when

$$
\max \left(\alpha_{1}, \beta_{1}\right) \leq 0 \quad \text { and } \quad \begin{cases}\alpha_{2} \geq 0 & \text { if } \alpha_{1}=0, \\ \beta_{2} \leq 0 & \text { if } \beta_{1}=0\end{cases}
$$

(ii) Let the parameters be given by (3.14). The embedding

$$
\operatorname{id}_{B}: B_{p_{1}, q_{1}}^{s_{1}}\left(\mathbb{R}^{n}, w_{(\boldsymbol{\alpha}, \boldsymbol{\beta})}\right) \hookrightarrow B_{p_{2}, q_{2}}^{s_{2}}\left(\mathbb{R}^{n}\right)
$$

is continuous if, and only if,

$$
\left\{\begin{array}{lll}
\text { either } & \frac{\beta_{1}}{p_{1}}>\frac{n}{p^{*}}, & \beta_{2} \in \mathbb{R}, \\
\text { or } & \frac{\beta_{1}}{p_{1}}=\frac{n}{p^{*}}, & \frac{\beta_{2}}{p_{1}}>\frac{1}{p^{*}} \quad \text { if } \quad p^{*}<\infty \\
& \beta_{1}=0, & \beta_{2} \geq 0 \quad \text { if } \quad p^{*}=\infty
\end{array}\right.
$$


and one of the following conditions is satisfied,

$$
\left\{2^{-\nu\left(\delta-\frac{\alpha_{1}}{p_{1}}\right)}(1+\nu)^{-\frac{\alpha_{2}}{p_{1}}}\right\}_{\nu} \in \ell_{q^{*}} \quad \text { if } \quad \frac{\alpha_{1}}{p_{1}}>\frac{n}{p^{*}}, \alpha_{2} \in \mathbb{R},
$$

$$
\left\{2^{-\nu\left(\delta-\frac{n}{p^{*}}\right)}\right\}_{\nu} \in \ell_{q^{*}} \quad \text { if } \quad \frac{\alpha_{1}}{p_{1}}<\frac{n}{p^{*}}, \alpha_{2} \in \mathbb{R}, \quad \text { or } \quad \frac{\alpha_{1}}{p_{1}}=\frac{n}{p^{*}}, \frac{\alpha_{2}}{p_{1}}>\frac{1}{p^{*}},
$$

$$
\left\{2^{-\nu\left(\delta-\frac{n}{p^{*}}\right)}(1+\nu)^{\frac{1}{p^{*}}-\frac{\alpha_{2}}{p_{1}}}\right\}_{\nu} \in \ell_{q^{*}} \quad \text { if } \quad \frac{\alpha_{1}}{p_{1}}=\frac{n}{p^{*}}, \frac{\alpha_{2}}{p_{1}}<\frac{1}{p^{*}},
$$

$$
\left\{2^{-\nu\left(\delta-\frac{n}{p^{*}}\right)} \log ^{\frac{1}{p^{*}}}(1+\nu)\right\}_{\nu} \in \ell_{q^{*}} \quad \text { if } \quad \frac{\alpha_{1}}{p_{1}}=\frac{n}{p^{*}}, \frac{\alpha_{2}}{p_{1}}=\frac{1}{p^{*}} .
$$

(iii) The embedding (3.27) is compact if, and only if,

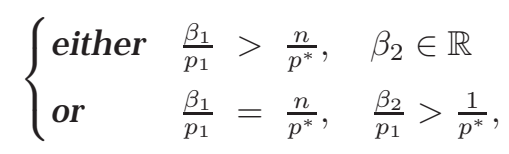

and

$$
\begin{cases}\text { either } & \delta>\max \left(\frac{\alpha_{1}}{p_{1}}, \frac{n}{p^{*}}\right), \quad \alpha_{2} \in \mathbb{R}, \\ \text { or } & \delta=\frac{\alpha_{1}}{p_{1}}>\frac{n}{p^{*}}, \quad \frac{\alpha_{2}}{p_{1}}>\frac{1}{q^{*}} .\end{cases}
$$

Proof. Step 1. Part (i) can be proved similar to [25, Prop. 2.6], cf. [14, Lemma 2.3]. If $w \in \mathcal{A}_{1}$ and a function $b$ is positive a.e. and $b, b^{-1} \in L_{\infty}\left(\mathbb{R}^{n}\right)$, then $b w \in \mathcal{A}_{1}$. So if a locally integrable, positive a.e. function is equivalent to an $\mathcal{A}_{1}$ weight it is also an $\mathcal{A}_{1}$ weight. Let

$w_{1}(x)=\left\{\begin{array}{ll}1, & |x| \leq 1, \\ (1+|\log | x||)^{\gamma_{1}}, & |x|>1,\end{array} \quad w_{2}(x)= \begin{cases}1, & |x|>1, \\ (1+|\log | x||)^{\gamma_{2},} & |x| \leq 1 .\end{cases}\right.$

Then $w_{1}(x) \sim \log (2+|x|)^{\gamma_{1}}, \gamma_{1} \in \mathbb{R}$, and $w_{2}(x) \sim \log \left(2+|x|^{-1}\right)^{\gamma_{2}}$, $\gamma_{2} \in \mathbb{R}$, so $w_{1}$ and $w_{2}$ are $\mathcal{A}_{1}$ weights if $\gamma_{1} \leq 0$ and $\gamma_{2} \geq 0$, see Example 2.3. In a similar way $w_{3}(x)=\max \left(1,|x|^{\gamma_{3}}(1+|\log | x||)^{\gamma_{4}}\right)$ and $w_{4}(x)=\min \left(1,|x|^{\gamma_{5}}(1+|\log | x||)^{\gamma_{6}}\right)$ are $\mathcal{A}_{1}$ weights if $\gamma_{3}, \gamma_{5}<0$ and $\gamma_{4}, \gamma_{6} \in \mathbb{R}$. Consequently, $w_{(\boldsymbol{\alpha}, \boldsymbol{\beta})}(x) \sim w_{i}^{\theta}(x) w_{j}^{1-\theta}(x)$, for suitably 
chosen $i, j$ and $\gamma_{1}, \ldots, \gamma_{6}$. Hence $w_{(\boldsymbol{\alpha}, \boldsymbol{\beta})} \in \mathcal{A}_{1}$.

Concerning (ii) and (iii) we apply Proposition 3.3(ii), (iii) with $w_{1}=w_{(\boldsymbol{\alpha}, \boldsymbol{\beta})}$ and $w_{2} \equiv 1$; hence

$$
w_{1}\left(Q_{\nu, m}\right) \sim 2^{-\nu n}\left\{\begin{array}{lrr}
2^{-\nu \alpha_{1}}(1+\nu)^{\alpha_{2}} & \text { if } & m=0, \\
\left|2^{-\nu} m\right|^{\alpha_{1}}\left(1-\log \left|2^{-\nu} m\right|\right)^{\alpha_{2}} & \text { if } & 1 \leq|m|<2^{\nu}, \\
\left|2^{-\nu} m\right|^{\beta_{1}}\left(1+\log \left|2^{-\nu} m\right|\right)^{\beta_{2}} & \text { if } & |m| \geq 2^{\nu},
\end{array}\right.
$$

and $w_{2}\left(Q_{\nu, m}\right) \sim 2^{-\nu n}, \nu \in \mathbb{N}_{0}, m \in \mathbb{Z}^{n}$. This leads to

$$
\frac{w_{2}\left(Q_{\nu, m}\right)^{1 / p_{2}}}{w_{1}\left(Q_{\nu, m}\right)^{1 / p_{1}}} \sim 2^{-\nu\left(\frac{n}{p_{2}}-\frac{n}{p_{1}}\right)} d_{m}^{\nu}, \quad \nu \in \mathbb{N}_{0}, \quad m \in \mathbb{Z}^{n}
$$

with

$$
d_{m}^{\nu}:=\left\{\begin{array}{llr}
2^{\nu \frac{\alpha_{1}}{p_{1}}}(1+\nu)^{-\frac{\alpha_{2}}{p_{1}}} & \text { if } & m=0, \\
\left|2^{-\nu} m\right|^{-\frac{\alpha_{1}}{p_{1}}}\left(1-\log \left|2^{-\nu} m\right|\right)^{-\frac{\alpha_{2}}{p_{1}}} & \text { if } & 1 \leq|m|<2^{\nu}, \\
\left|2^{-\nu} m\right|^{-\frac{\beta_{1}}{p_{1}}}\left(1+\log \left|2^{-\nu} m\right|\right)^{-\frac{\beta_{2}}{p_{1}}} & \text { if } & |m| \geq 2^{\nu} .
\end{array}\right.
$$

Then (3.9) can be rewritten as

$$
\left\{2^{-\nu \delta}\left\|\left\{d_{m}^{\nu}\right\}_{m} \mid \ell_{p^{*}}\right\|\right\}_{\nu} \in \ell_{q^{*}} .
$$

Step 2. Let $\nu \in \mathbb{N}_{0}$ be fixed for the moment; we study $\left\|\left\{d_{m}^{\nu}\right\}_{m} \mid \ell_{p^{*}}\right\|$ and split it into

$$
\begin{aligned}
& D_{1}^{\nu}=\left\|\left\{d_{m}^{\nu}\right\}_{m=0} \mid \ell_{p^{*}}\right\|=d_{0}^{\nu}, \\
& D_{2}^{\nu}=\left\|\left\{d_{m}^{\nu}\right\}_{m, 1 \leq|m|<2^{\nu}} \mid \ell_{p^{*}}\right\|, \\
& D_{3}^{\nu}=\left\|\left\{d_{m}^{\nu}\right\}_{m,|m| \geq 2^{\nu}} \mid \ell_{p^{*}}\right\|
\end{aligned}
$$

in obvious notation. Consequently, $\left\|\left\{d_{m}^{\nu}\right\}_{m} \mid \ell_{p^{*}}\right\| \sim \max \left(D_{1}^{\nu}, D_{2}^{\nu}, D_{3}^{\nu}\right) \sim$ $D_{1}^{\nu}+D_{2}^{\nu}+D_{3}^{\nu}$. Firstly, (3.37) and (3.39) give

$$
D_{1}^{\nu} \sim 2^{\nu \frac{\alpha_{1}}{p_{1}}}(1+\nu)^{-\frac{\alpha_{2}}{p_{1}}}, \quad p^{*} \leq \infty .
$$

Next we consider $D_{3}^{\nu}$ and obtain for $p^{*}=\infty$ that

$$
D_{3}^{\nu} \sim \sup _{|y| \geq 1}|y|^{-\frac{\beta_{1}}{p_{1}}}(1+\log |y|)^{-\frac{\beta_{2}}{p_{1}}} \leq c<\infty
$$


(independent of $\nu$ ) if, and only if,

$$
\text { either } \beta_{1}>0, \beta_{2} \in \mathbb{R}, \quad \text { or } \quad \beta_{1}=0, \beta_{2} \geq 0 \text {. }
$$

If $p^{*}<\infty$, then

$$
\begin{aligned}
D_{3}^{\nu} & \sim\left(\sum_{|m| \geq 2^{\nu}}\left|2^{-\nu} m\right|^{-\frac{\beta_{1}}{p_{1}} p^{*}}\left(1+\log \left|2^{-\nu} m\right|\right)^{-\frac{\beta_{2}}{p_{1}} p^{*}}\right)^{1 / p^{*}} \\
& \sim\left(\sum_{l=\nu}^{\infty} \sum_{|m| \sim 2^{l}}\left|2^{-\nu} m\right|^{-\frac{\beta_{1}}{p_{1}} p^{*}}\left(1+\log \left|2^{-\nu} m\right|\right)^{-\frac{\beta_{2}}{p_{1}} p^{*}}\right)^{1 / p^{*}} \\
& \sim\left(\sum_{l=\nu}^{\infty} 2^{l n} 2^{-(l-\nu) \frac{\beta_{1}}{p_{1}} p^{*}}(1+l-\nu)^{-\frac{\beta_{2}}{p_{1}} p^{*}}\right)^{1 / p^{*}} \\
& \sim 2^{\nu \frac{n}{p^{*}}}\left(\sum_{k=1}^{\infty} 2^{-k\left(\frac{\beta_{1}}{p_{1}}-\frac{n}{p^{*}}\right) p^{*}} k^{-\frac{\beta_{2}}{p_{1}} p^{*}}\right)^{1 / p^{*}}
\end{aligned}
$$

such that Lemma 3.8 implies $D_{3}^{\nu}<\infty$ if, and only if,

$$
\text { either } \frac{\beta_{1}}{p_{1}}>\frac{n}{p^{*}}, \beta_{2} \in \mathbb{R}, \quad \text { or } \quad \frac{\beta_{1}}{p_{1}}=\frac{n}{p^{*}}, \frac{\beta_{2}}{p_{1}}>\frac{1}{p^{*}} .
$$

Now (3.43) and (3.44) give (3.28) and

$$
D_{3}^{\nu} \sim 2^{\nu \frac{n}{p^{*}}}, \quad p^{*} \leq \infty .
$$

It remains to consider $D_{2}^{\nu}$. For $p^{*}=\infty$ one immediately verifies (3.46) $D_{2}^{\nu} \sim \sup _{1 \leq|m|<2^{\nu}}\left|2^{-\nu} m\right|^{-\frac{\alpha_{1}}{p_{1}}}\left(1-\log \left|2^{-\nu} m\right|\right)^{-\frac{\alpha_{2}}{p_{1}}} \sim \max \left(D_{1}^{\nu}, D_{3}^{\nu}\right)$.

If $p^{*}<\infty$, then

$$
\begin{aligned}
D_{2}^{\nu} & \sim\left(\sum_{1 \leq|m|<2^{\nu}}\left|2^{-\nu} m\right|^{-\frac{\alpha_{1}}{p_{1}} p^{*}}\left(1-\log \left|2^{-\nu} m\right|\right)^{-\frac{\alpha_{2}}{p_{1}} p^{*}}\right)^{1 / p^{*}} \\
& \sim\left(\sum_{l=0}^{\nu} \sum_{|m| \sim 2^{l}}\left|2^{-\nu} m\right|^{-\frac{\alpha_{1}}{p_{1}} p^{*}}\left(1-\log \left|2^{-\nu} m\right|\right)^{-\frac{\alpha_{2}}{p_{1}} p^{*}}\right)^{1 / p^{*}}
\end{aligned}
$$




$$
\begin{aligned}
& \sim\left(\sum_{l=0}^{\nu} 2^{l n} 2^{(\nu-l) \frac{\alpha_{1}}{p_{1}} p^{*}}(1+\nu-l)^{-\frac{\alpha_{2}}{p_{1}} p^{*}}\right)^{1 / p^{*}} \\
& \sim 2^{\nu \frac{n}{p^{*}}}\left(\sum_{k=1}^{\nu+1} 2^{k\left(\frac{\alpha_{1}}{p_{1}}-\frac{n}{p^{*}}\right) p^{*}} k^{-\frac{\alpha_{2}}{p_{1}} p^{*}}\right)^{1 / p^{*}}
\end{aligned}
$$

such that Lemma 3.8 implies

$$
D_{2}^{\nu} \sim 2^{\nu \frac{\alpha_{1}}{p_{1}}}(1+\nu)^{-\frac{\alpha_{2}}{p_{1}}} \quad \text { if } \quad \frac{\alpha_{1}}{p_{1}}>\frac{n}{p^{*}}, \alpha_{2} \in \mathbb{R},
$$

$$
D_{2}^{\nu} \sim 2^{\nu \frac{n}{p^{*}}} \quad \text { if } \quad \frac{\alpha_{1}}{p_{1}}<\frac{n}{p^{*}}, \alpha_{2} \in \mathbb{R}, \quad \text { or } \quad \frac{\alpha_{1}}{p_{1}}=\frac{n}{p^{*}}, \frac{\alpha_{2}}{p_{1}}>\frac{1}{p^{*}},
$$

$$
D_{2}^{\nu} \sim 2^{\nu \frac{n}{p^{*}}}(1+\nu)^{\frac{1}{p^{*}}-\frac{\alpha_{2}}{p_{1}}} \quad \text { if } \quad \frac{\alpha_{1}}{p_{1}}=\frac{n}{p^{*}}, \frac{\alpha_{2}}{p_{1}}<\frac{1}{p^{*}},
$$

$$
D_{2}^{\nu} \sim 2^{\nu \frac{n}{p^{*}}} \log \frac{1}{p^{*}}(1+\nu) \quad \text { if } \quad \frac{\alpha_{1}}{p_{1}}=\frac{n}{p^{*}}, \frac{\alpha_{2}}{p_{1}}=\frac{1}{p^{*}} .
$$

Summarising, (3.42), (3.45) and (3.46) give

$$
\left\|\left\{d_{m}^{\nu}\right\}_{m} \mid \ell_{p^{*}}\right\| \sim \max \left(2^{\nu \frac{\alpha_{1}}{p_{1}}}(1+\nu)^{-\frac{\alpha_{2}}{p_{1}}}, 2^{\nu \frac{n}{p^{*}}}\right)
$$

for $p^{*}=\infty$. In view of (3.47), (3.48) we also arrive at (3.51) in case of $p^{*}<\infty$ and

$$
\frac{\alpha_{1}}{p_{1}} \neq \frac{n}{p^{*}}, \alpha_{2} \in \mathbb{R}, \quad \text { or } \quad \frac{\alpha_{1}}{p_{1}}=\frac{n}{p^{*}}, \frac{\alpha_{2}}{p_{1}}>\frac{1}{p^{*}} .
$$

Consequently, for $p^{*} \leq \infty$ and $\nu \geq \nu_{0}$,

$$
\left\|\left\{d_{m}^{\nu}\right\}_{m} \mid \ell_{p^{*}}\right\| \sim 2^{\nu \frac{\alpha_{1}}{p_{1}}}(1+\nu)^{-\frac{\alpha_{2}}{p_{1}}} \quad \text { if } \quad \frac{\alpha_{1}}{p_{1}}>\frac{n}{p^{*}}, \alpha_{2} \in \mathbb{R}
$$

$$
\left\|\left\{d_{m}^{\nu}\right\}_{m} \mid \ell_{p^{*}}\right\| \sim 2^{\nu \frac{n}{p^{*}}} \quad \text { if } \quad \frac{\alpha_{1}}{p_{1}}<\frac{n}{p^{*}}, \alpha_{2} \in \mathbb{R}, \text { or } \frac{\alpha_{1}}{p_{1}}=\frac{n}{p^{*}}, \frac{\alpha_{2}}{p_{1}}>\frac{1}{p^{*}},
$$


whereas (3.42), (3.45) and (3.49) lead to

$$
\left\|\left\{d_{m}^{\nu}\right\}_{m} \mid \ell_{p^{*}}\right\| \sim 2^{\nu \frac{n}{p^{*}}}(1+\nu)^{\frac{1}{p^{*}}-\frac{\alpha_{2}}{p_{1}}} \quad \text { if } \quad \frac{\alpha_{1}}{p_{1}}=\frac{n}{p^{*}}, \frac{\alpha_{2}}{p_{1}}<\frac{1}{p^{*}},
$$

and

$$
\left\|\left\{d_{m}^{\nu}\right\}_{m} \mid \ell_{p^{*}}\right\| \sim 2^{\nu \frac{n}{p^{*}}} \log \frac{1}{p^{*}}(1+\nu) \quad \text { if } \quad \frac{\alpha_{1}}{p_{1}}=\frac{n}{p^{*}}, \frac{\alpha_{2}}{p_{1}}=\frac{1}{p^{*}},
$$

in view of (3.42), (3.45) and (3.50). Now (3.38) together with (3.53)(3.56) prove (3.29)-(3.32), respectively.

Step 3. It remains to prove (iii). Inspecting Proposition 3.3(iii), in particular (3.10), gives us the additional condition

$$
\lim _{\nu \rightarrow \infty} 2^{-\nu \delta}\left\|\left\{d_{m}^{\nu}\right\}_{m} \mid \ell_{p^{*}}\right\|=0 \quad \text { if } \quad q^{*}=\infty
$$

in view of (3.36). But (3.53)-(3.56) imply

$$
\text { either } \delta>\max \left(\frac{\alpha_{1}}{p_{1}}, \frac{n}{p^{*}}\right), \alpha_{2} \in \mathbb{R}, \quad \text { or } \quad \delta=\frac{\alpha_{1}}{p_{1}}>\frac{n}{p^{*}}, \alpha_{2}>0
$$

in that case. Together with the case $q^{*}<\infty$ from (ii) this is just (3.34). Likewise (3.11) requires

$$
\lim _{|m| \rightarrow \infty} \frac{w_{1}\left(Q_{\nu, m}\right)^{1 / p_{1}}}{w_{2}\left(Q_{\nu, m}\right)^{1 / p_{2}}}=\infty \quad \text { for all } \nu \in \mathbb{N}_{0} \quad \text { if } \quad p^{*}=\infty,
$$

but in view of (3.36), (3.37) this is nothing else than (3.33) for $p^{*}=\infty$.

Remark 3.10. It is sometimes more convenient to simplify conditions (3.29)-(3.32) in dependence on $q^{*}<\infty$ or $q^{*}=\infty$, respectively (and thus parallel to (3.28) in some sense).

If $q^{*}<\infty$, then (3.27) if, and only if, (3.28) and

$$
\text { either } \delta>\max \left(\frac{\alpha_{1}}{p_{1}}, \frac{n}{p^{*}}\right), \alpha_{2} \in \mathbb{R}, \quad \text { or } \quad \delta=\frac{\alpha_{1}}{p_{1}}>\frac{n}{p^{*}}, \frac{\alpha_{2}}{p_{1}}>\frac{1}{q^{*}} \text {. }
$$


If $q^{*}=\infty$, then (3.27) if, and only if, (3.28) and (3.60)

either $\delta>\max \left(\frac{\alpha_{1}}{p_{1}}, \frac{n}{p^{*}}\right), \alpha_{2} \in \mathbb{R}, \quad$ or $\quad \delta= \begin{cases}\frac{\alpha_{1}}{p_{1}}>\frac{n}{p^{*}}, & \alpha_{2} \geq 0, \\ \frac{n}{p^{*}}>\frac{\alpha_{1}}{p_{1}}, & \alpha_{2} \in \mathbb{R}, \\ \frac{n}{p^{*}}=\frac{\alpha_{1}}{p_{1}}, & \frac{\alpha_{2}}{p_{1}}>\frac{1}{p^{*}}, \\ \frac{n}{p^{*}}=\frac{\alpha_{1}}{p_{1}}=\frac{\alpha_{2}}{p_{1}}=0 .\end{cases}$

In particular, for $s_{1}=s_{2}=s, r \leq p, q_{1}=q_{2}=q$, hence $q^{*}=\infty$, $\delta=\frac{n}{p^{*}}=\frac{n}{r}-\frac{n}{p} \geq 0$, we obtain

$$
B_{p, q}^{s}\left(\mathbb{R}^{n}, w_{(\boldsymbol{\alpha}, \boldsymbol{\beta})}\right) \hookrightarrow B_{r, q}^{s}\left(\mathbb{R}^{n}\right)
$$

if

$$
\left\{\begin{array} { l l } 
{ \text { either } } & { \frac { \alpha _ { 1 } } { n p } < \frac { 1 } { r } - \frac { 1 } { p } , \alpha _ { 2 } \in \mathbb { R } , } \\
{ \text { or } } & { \frac { \alpha _ { 1 } } { n p } = \frac { 1 } { r } - \frac { 1 } { p } , \frac { \alpha _ { 2 } } { p } > \frac { 1 } { r } - \frac { 1 } { p } , }
\end{array} \text { and } \left\{\begin{array}{ll}
\text { either } & \frac{\beta_{1}}{n p}>\frac{1}{r}-\frac{1}{p}, \beta_{2} \in \mathbb{R}, \\
\text { or } & \frac{\beta_{1}}{n p}=\frac{1}{r}-\frac{1}{p}, \frac{\beta_{2}}{p}>\frac{1}{r}-\frac{1}{p} .
\end{array}\right.\right.
$$

There is never a compact embedding of type (3.61).

In the usual $\left(\frac{1}{p}, s\right)$-diagram, where any (weighted or unweighted) space of type $A_{p, q}^{s}$ is characterised by its parameters $s$ and $p$ (neglecting $q$ for the moment), we have sketched in the figure below the admitted area for the target space $B_{p_{2}, q_{2}}^{s_{2}}\left(\mathbb{R}^{n}\right)$ in order to obtain a continuous embedding $\operatorname{id}_{B}$ for fixed source space $B_{p_{1}, q_{1}}^{s_{1}}\left(\mathbb{R}^{n}, w_{(\boldsymbol{\alpha}, \boldsymbol{\beta})}\right)$. For convenience we have chosen the case $0<\alpha_{1}<\beta_{1}$, but the modifications in other cases should be obvious. We shall return to this figure below.

There is a counterpart of Proposition 3.9 in the double-weighted situation, that is, in the spirit of Remark 3.7. However, for convenience we formulate this result only in the special situation when $s_{1}=s_{2}, p_{1}=p_{2}$, and $q_{1}=q_{2}$.

Corollary 3.11. Let $w_{(\boldsymbol{\alpha}, \boldsymbol{\beta})}, w_{(\gamma, \varkappa)}$ be weights of type (3.21) with (3.22), respectively. Let $s \in \mathbb{R}, 0<p<\infty, 0<q \leq \infty$. Then

$$
B_{p, q}^{s}\left(\mathbb{R}^{n}, w_{(\boldsymbol{\alpha}, \boldsymbol{\beta})}\right) \hookrightarrow B_{p, q}^{s}\left(\mathbb{R}^{n}, w_{(\boldsymbol{\gamma}, \boldsymbol{\varkappa})}\right)
$$

if, and only if,

$$
\text { either } \alpha_{1}<\gamma_{1}, \alpha_{2} \in \mathbb{R}, \gamma_{2} \in \mathbb{R}, \quad \text { or } \quad \alpha_{1}=\gamma_{1}, \alpha_{2} \geq \gamma_{2} \text {, }
$$




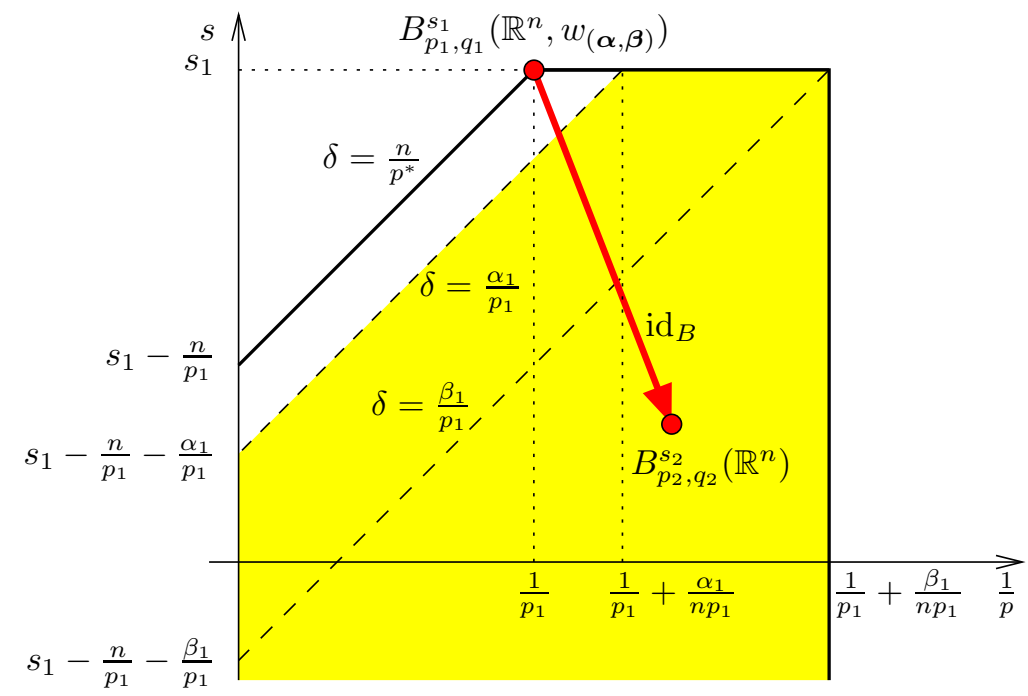

FiguRE 1. Possible embeddings in case of $0<\alpha_{1}<\beta_{1}$

and

(3.65) either $\beta_{1}>\varkappa_{1}, \beta_{2} \in \mathbb{R}, \varkappa_{2} \in \mathbb{R}, \quad$ or $\beta_{1}=\varkappa_{1}, \beta_{2} \geq \varkappa_{2}$.

Proof. Note that $p^{*}=q^{*}=\infty, \delta=\frac{n}{p^{*}}=0$. An application of (3.28), (3.60) to (3.61) with $B_{p, q}^{s}\left(\mathbb{R}^{n}, w_{(\boldsymbol{\alpha}-\boldsymbol{\gamma}, \boldsymbol{\beta}-\varkappa)} \hookrightarrow B_{p, q}^{s}\left(\mathbb{R}^{n}\right)\right)$ gives the result.

We now come to some limiting embedding result adapted to our weight $w_{(\boldsymbol{\alpha}, \boldsymbol{\beta})}$.

Proposition 3.12. Let $0<p<\infty, 0<q \leq \infty, 0<r \leq p, u \in \mathbb{R}$, and $w_{(\boldsymbol{\alpha}, \boldsymbol{\beta})}$ given by (3.21) with $\alpha_{1}>-n, \beta_{1}>0, \max \left(\alpha_{1}, 0\right) \leq \beta_{1}$, $\alpha_{2}, \beta_{2} \in \mathbb{R}$. Then

$$
A_{p, q}^{s}\left(\mathbb{R}^{n}, w_{(\boldsymbol{\alpha}, \boldsymbol{\beta})}\right) \hookrightarrow A_{q}^{s}\left(L_{r, p}(\log L)_{u}\right)\left(\mathbb{R}^{n}\right)
$$

if

(3.67)

$$
\begin{cases}u \in \mathbb{R}, & \text { if } \frac{1}{p}+\frac{\max \left(\alpha_{1}, 0\right)}{n p}<\frac{1}{r}<\frac{1}{p}+\frac{\beta_{1}}{n p}, \\ u \leq \frac{\alpha_{2}}{p}, & \text { if } \quad \frac{1}{p}+\frac{\max \left(\alpha_{1}, 0\right)}{n p}=\frac{1}{r} \quad \text { and } \alpha_{1}>0, \text { or } \alpha_{1}=0, \alpha_{2}<0, \\ u \leq 0, & \text { if } \quad \frac{1}{p}+\frac{\max \left(\alpha_{1}, 0\right)}{n p}=\frac{1}{r} \quad \text { and } \alpha_{1}<0, \text { or } \alpha_{1}=0, \alpha_{2} \geq 0, \\ u \leq \frac{\beta_{2}}{p}, & \text { if } \quad \frac{1}{p}+\frac{\beta_{1}}{n p}=\frac{1}{r} .\end{cases}
$$


Proof. In view of Definition 2.6 it is sufficient to prove

$$
L_{p}\left(\mathbb{R}^{n}, w_{(\boldsymbol{\alpha}, \boldsymbol{\beta})}\right) \hookrightarrow L_{r, p}(\log L)_{u}\left(\mathbb{R}^{n}\right)
$$

whenever (3.67) is satisfied. This result may be well-known, but can be proved easily by interpolation arguments similar to [22, Lemma 3.33] using real interpolation with function parameter as presented in [34, Example 3, p. 187] together with Hölder's inequality, see also [23, Lemma 3.3]. Thus the classical Hölder inequality can be extended to

$$
\left\|f g\left|L_{r, p}(\log L)_{u}\left(\mathbb{R}^{n}\right)\|\leq c\| f\right| L_{p}\left(\mathbb{R}^{n}\right)\right\|\left\|g \mid L_{v, \infty}(\log L)_{u}\left(\mathbb{R}^{n}\right)\right\|,
$$

where $\frac{1}{r}=\frac{1}{p}+\frac{1}{v}, u \in \mathbb{R}$. In order to verify (3.68) under the assumptions (3.67) it is thus sufficient to show that

$$
\left(w_{(\boldsymbol{\alpha}, \boldsymbol{\beta})}\right)^{-\frac{1}{p}} \in L_{v, \infty}(\log L)_{u}\left(\mathbb{R}^{n}\right)
$$

with $\frac{1}{v}=\frac{1}{r}-\frac{1}{p}$ and $u$ according to (3.67), since taking this for granted one may continue (3.69) by

$$
\begin{aligned}
\left\|f \mid L_{r, p}(\log L)_{u}\left(\mathbb{R}^{n}\right)\right\| & \leq c_{1}\left\|f\left(w_{(\boldsymbol{\alpha}, \boldsymbol{\beta})}\right)^{\frac{1}{p}}\left|L_{p}\left(\mathbb{R}^{n}\right)\|\|\left(w_{(\boldsymbol{\alpha}, \boldsymbol{\beta})}\right)^{-\frac{1}{p}}\right| L_{v, \infty}(\log L)_{u}\left(\mathbb{R}^{n}\right)\right\| \\
& \leq c_{2}\left\|f \mid L_{p}\left(\mathbb{R}^{n}, w_{(\boldsymbol{\alpha}, \boldsymbol{\beta})}\right)\right\|
\end{aligned}
$$

and obtains (3.68). We verify (3.70). Note that for $\alpha_{1}>0$ or $\alpha_{1}=0$, $\alpha_{2}<0$ we obtain

$$
\left[\left(w_{(\boldsymbol{\alpha}, \boldsymbol{\beta})}\right)^{-\frac{1}{p}}\right]^{*}(t) \sim\left\{\begin{array}{lll}
t^{-\frac{\alpha_{1}}{n_{p}}}(1+|\log t|)^{-\frac{\alpha_{2}}{p}} & \text { if } t \leq 1, \\
t^{-\frac{\beta_{1}}{n p}}(1+|\log t|)^{-\frac{\beta_{2}}{p}} & \text { if } \quad t>1,
\end{array}\right.
$$

and the definition (2.15) of $L_{v, \infty}(\log L)_{u}\left(\mathbb{R}^{n}\right)$ leads to (3.70) if, and only if,

$$
\sup _{0<t<1} t^{\frac{1}{r}-\frac{1}{p}-\frac{\alpha_{1}}{n p}}(1+|\log t|)^{u-\frac{\alpha_{2}}{p}}+\sup _{t>1} t^{\frac{1}{r}-\frac{1}{p}-\frac{\beta_{1}}{n p}}(1+|\log t|)^{u-\frac{\beta_{2}}{p}}
$$

is finite. But this covers the first, second and last line in (3.67). In case of $\alpha_{1}<0$ or $\alpha_{1}=0, \alpha_{2} \geq 0$, (3.71) has to be replaced by

$$
\left[\left(w_{(\boldsymbol{\alpha}, \boldsymbol{\beta})}\right)^{-\frac{1}{p}}\right]^{*}(t) \sim \begin{cases}1 & \text { if } t \rightarrow 0, \\ t^{-\frac{\beta_{1}}{n p}}(1+|\log t|)^{-\frac{\beta_{2}}{p}} & \text { if } t \rightarrow \infty,\end{cases}
$$


such that the counterpart of (3.72),

$$
\sup _{0<t<1} t^{\frac{1}{r}-\frac{1}{p}}(1+|\log t|)^{u}+\sup _{t>1} t^{\frac{1}{r}-\frac{1}{p}-\frac{\beta_{1}}{n p}}(1+|\log t|)^{u-\frac{\beta_{2}}{p}}
$$

results in the third line and concludes the proof.

Remark 3.13. Plainly, if $\alpha_{1}=\beta_{1}>0$ in Proposition 3.12, then (3.67) is to be understood such that both the second and last line hold, i.e., we have embedding (3.66) in this case if $\frac{1}{r}=\frac{1}{p}+\frac{\beta_{1}}{n p}=$ $\frac{1}{p}+\frac{\alpha_{1}}{n p}>\frac{1}{p}$ and $u \leq \frac{\min \left(\alpha_{2}, \beta_{2}\right)}{p}$. Moreover, since for $r<p$ we have

$$
L_{r, p}(\log L)_{u}\left(\mathbb{R}^{n}\right) \hookrightarrow L_{r}\left(\mathbb{R}^{n}\right) \quad \text { if } \quad u>\frac{1}{r}-\frac{1}{p}>0,
$$

cf. [1, Thm. 9.3], Proposition 3.12 refines our result (3.61) with (3.62). We complement our above Figure 1 in view of Proposition 3.66 .

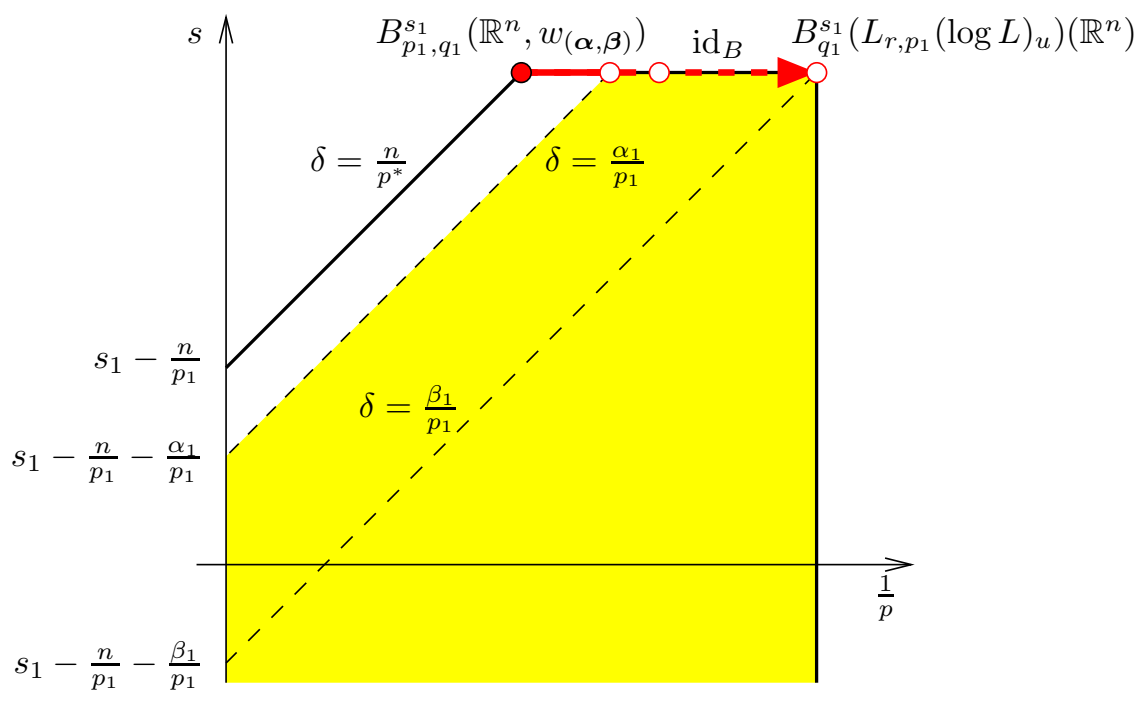

FIGURE 2. The borderline case $s_{1}=s_{2}$

Remark 3.14. In [25] we dealt with weights of type (3.15) which can be regarded as special cases of (3.21) with $w_{(\boldsymbol{\alpha}, \boldsymbol{\beta})}=w_{\alpha_{1}, \beta_{1}}$ if $\alpha_{2}=\beta_{2}=0$. Using, in addition, our notation (2.16), we proved 
in [25, Prop. 2.10] that

$$
B_{p, q}^{s}\left(\mathbb{R}^{n}, w_{(\boldsymbol{\alpha}, \boldsymbol{\beta})}\right) \hookrightarrow B_{q}^{s}\left(L_{r, p}(\log L)_{0}\right)\left(\mathbb{R}^{n}\right), \quad \frac{1}{r}=\frac{1}{p}+\frac{\beta_{1}}{n p},
$$

hence a special case of Proposition 3.12. (Note that we formulated the result in [25, Prop. 2.10] slightly weaker than our proof would allow.)

We end this section with some compactness result for $F$-spaces. For that purpose we briefly recall some endpoint observation by EDMUNDS and NeTRUSOv in their paper [12]: let $\Omega \subset \mathbb{R}^{n}$ be bounded, say, the open unit cube in $\mathbb{R}^{n}$ (as in [12]), and the spaces $F_{q}^{s}\left(L_{p, p}(\log L)_{a}\right)(\Omega)$ obtained by restriction from their $\mathbb{R}^{n}$ counterparts in Definition 2.6(ii), quasi-normed in the usual way. Then [12, Thm. 4.1] implies (in our notation) that for $a_{1}, a_{2} \in \mathbb{R}$ with $a_{1}>a_{2}, 0<p_{1}, p_{2}<\infty, 0<q_{1}, q_{2} \leq \infty, s_{1}, s_{2} \in \mathbb{R}$ with $s_{1}>s_{2}$ and

$$
\delta=s_{1}-s_{2}-\frac{n}{p_{1}}+\frac{n}{p_{2}}=0
$$

then

$$
\operatorname{id}_{F, \log }^{\Omega}: F_{q_{1}}^{s_{1}}\left(L_{p_{1}, p_{1}}(\log L)_{a_{1}}\right)(\Omega) \hookrightarrow F_{q_{2}}^{s_{2}}\left(L_{p_{2}, p_{2}}(\log L)_{a_{2}}\right)(\Omega)
$$

is compact. Of course, (3.76) with $a_{1}=a_{2}=0$ is the well-known (continuous, but not compact) embedding of $F$-spaces, see also (3.6) for its weighted generalisation (on $\mathbb{R}^{n}$ ).

Corollary 3.15. Let $w_{(\boldsymbol{\alpha}, \boldsymbol{\beta})}$ be given by (3.21), (3.22), and

$$
\operatorname{id}_{F}: F_{p_{1}, q_{1}}^{s_{1}}\left(\mathbb{R}^{n}, w_{(\boldsymbol{\alpha}, \boldsymbol{\beta})}\right) \hookrightarrow F_{p_{2}, q_{2}}^{s_{2}}\left(\mathbb{R}^{n}\right),
$$

where the parameters are given by (3.14) with $p_{2}<\infty$.

(i) Let (3.77) be compact. Then we have (3.33) and

$$
\left\{\begin{array}{lll}
\text { either } & \delta>\max \left(\frac{\alpha_{1}}{p_{1}}, \frac{n}{p^{*}}\right), & \alpha_{2} \in \mathbb{R}, \\
\text { or } & \delta=\frac{\alpha_{1}}{p_{1}}>\frac{n}{p^{*}}, & \frac{\alpha_{2}}{p_{1}}>\frac{1}{p^{*}} .
\end{array}\right.
$$

(ii) Assume that (3.33) and either

$$
\delta>\max \left(\frac{\alpha_{1}}{p_{1}}, \frac{n}{p^{*}}\right) \quad \text { and } \quad \alpha_{2} \in \mathbb{R}
$$


(3.80)

$$
\delta=\frac{\alpha_{1}}{p_{1}}>\frac{n}{p^{*}} \quad \text { and } \begin{cases}\frac{\alpha_{2}}{p_{1}}>\frac{1}{p^{*}}, & \text { if } p_{1}<p_{2}, \\ \frac{\alpha_{2}}{p_{1}}>\min \left(\frac{\delta}{n}, \frac{1}{\min \left(p_{2}, q_{2}\right)}-\frac{1}{\max \left(p_{1}, q_{1}\right)}\right), & \text { if } p_{1} \geq p_{2},\end{cases}
$$

are satisfied. Then (3.77) is compact.

Proof. Step 1. In view of the embeddings (3.2) and Proposition 3.9(iii) all that is left to verify concerns the limiting situations when $\delta=\frac{\alpha_{1}}{p_{1}}>\frac{n}{p^{*}}$; in particular, we have to deal with $\alpha_{1}>0$ now. Note that the conditions for $\alpha_{2}$ in case of $p_{1} \geq p_{2}$ in (3.80) are more restrictive than their counterpart in (3.78), since

$$
\min \left(\frac{\delta}{n}, \frac{1}{\min \left(p_{2}, q_{2}\right)}-\frac{1}{\max \left(p_{1}, q_{1}\right)}\right) \geq \frac{1}{p_{2}}-\frac{1}{p_{1}}=\frac{1}{p^{*}} .
$$

Step 2 . We begin with the necessity part (i) and have to show that the compactness of (3.77) leads to $\frac{\alpha_{2}}{p_{1}}>\frac{1}{p^{*}}$. Since $\alpha_{1}>0$ we can apply Proposition 3.1(iii) with $d=n+\alpha_{1}$ for $w=w_{(\boldsymbol{\alpha}, \boldsymbol{\beta})}$ and $d=n$ for $w \equiv 1$, and conclude from (3.77) the compactness of

$$
B_{r_{1}, p_{1}}^{\sigma_{1}}\left(\mathbb{R}^{n}, w_{(\boldsymbol{\alpha}, \boldsymbol{\beta})}\right) \hookrightarrow B_{r_{2}, p_{2}}^{\sigma_{2}}\left(\mathbb{R}^{n}\right)
$$

for suitably chosen $\sigma_{1}>s_{1} \geq s_{2}>\sigma_{2}, r_{1}<p_{1}, r_{2}>p_{2}$, with

$$
\sigma_{1}-\frac{n+\alpha_{1}}{r_{1}}=s_{1}-\frac{n+\alpha_{1}}{p_{1}}, \quad \sigma_{2}-\frac{n}{r_{2}}=s_{2}-\frac{n}{p_{2}} .
$$

Application of Proposition 3.9(iii) with

$$
\widetilde{\delta}=\sigma_{1}-\frac{n}{r_{1}}-\sigma_{2}+\frac{n}{r_{2}}=\delta-\frac{\alpha_{1}}{p_{1}}+\frac{\alpha_{1}}{r_{1}}=\frac{\alpha_{1}}{r_{1}}
$$

leads to $\frac{\alpha_{2}}{r_{1}}>\frac{1}{p^{*}}$ for arbitrarily chosen $r_{1}<p_{1}$. Hence $\frac{\alpha_{2}}{p_{1}} \geq \frac{1}{p^{*}}$ and it is sufficient to show that the compactness of the embedding (3.77) excludes the case $\frac{\alpha_{2}}{p_{1}}=\frac{1}{p^{*}}$. Assume first $p^{*}=\infty$, that is, $p_{1} \leq p_{2}$. Then the compactness of (3.77) and (3.2) imply the compactness of

$$
B_{p_{1}, \min \left(p_{1}, q_{1}\right)}^{s_{1}}\left(\mathbb{R}^{n}, w_{(\boldsymbol{\alpha}, \boldsymbol{\beta})}\right) \hookrightarrow B_{p_{2}, \max \left(p_{2}, q_{2}\right)}^{s_{2}}\left(\mathbb{R}^{n}\right),
$$

such that by Proposition 3.1(iii) the parameters satisfy (3.33), $\delta=$ $\frac{\alpha_{1}}{p_{1}}>0$, and thus by (3.34)

$$
\frac{\alpha_{2}}{p_{1}}>\left(\frac{1}{\max \left(p_{2}, q_{2}\right)}-\frac{1}{\min \left(p_{1}, q_{1}\right)}\right)_{+}=0
$$


excluding $\frac{\alpha_{2}}{p_{1}}=\frac{1}{p^{*}}=0$. Now let $p^{*}<\infty$ and $\frac{\alpha_{2}}{p_{1}}=\frac{1}{p^{*}}>0$. In this case we do not even have a continuous embedding of type (3.77) (not to speak about compactness). This is parallel to the $B$-situation when $\delta=\frac{\alpha_{1}}{p_{1}}>\frac{n}{p^{*}}$ and $\frac{\alpha_{2}}{p_{1}}=\frac{1}{q^{*}}>0$, see Remark 3.10. To see this we adapt an example from [42, Sect. 5.1] appropriately. Let a function $f$ be given by

$$
f=\sum_{j=0}^{\infty} a_{j}\left(\Delta^{N} \varphi\right)\left(2^{j}\left(\cdot-x^{j}\right)\right)
$$

where $a_{j} \in \mathbb{C}, N \in \mathbb{N}$ is sufficiently large, and $\varphi \in \mathcal{S}\left(\mathbb{R}^{n}\right)$ with compact support near the origin. The points $x^{j}$ are chosen such that the support of $\left(\Delta^{N} \varphi\right)\left(2^{j}\left(\cdot-x^{j}\right)\right)$ and $\left(\Delta^{N} \varphi\right)\left(2^{k}\left(\cdot-x^{k}\right)\right)$ are pairwise disjoint for $j \neq k$. According to [42, eq. (5.1.14)],

$$
\left\|f \mid F_{p_{2}, q_{2}}^{s_{2}}\left(\mathbb{R}^{n}\right)\right\| \sim\left(\sum_{m=0}^{\infty} 2^{m\left(s_{2}-\frac{n}{p_{2}}\right) p_{2}}\left|a_{m}\right|^{p_{2}}\right)^{1 / p_{2}} .
$$

On the other hand, repeating the same steps as in [42] for weighted spaces, now using the corresponding characterisations by local means from [6,7] (in the version recalled in [24, Thm. 3.5]), we obtain

$$
\left\|f \mid F_{p_{1}, q_{1}}^{s_{1}}\left(\mathbb{R}^{n}, w_{(\boldsymbol{\alpha}, \boldsymbol{\beta})}\right)\right\| \leq c\left(\sum_{m=0}^{\infty} 2^{m\left(s_{1}-\frac{n}{p_{1}}\right) p_{1}} 2^{-m \alpha_{1}}(1+m)^{\alpha_{2}}\left|a_{m}\right|^{p_{1}}\right)^{1 / p_{1}} .
$$

Let $f_{\gamma}$ be given by (3.83) with $a_{m}$ replaced by

$$
a_{m}^{\gamma}=2^{-m\left(s_{2}-\frac{n}{p_{2}}\right)}(1+m)^{-\frac{1}{p_{2}}}(1+\log (1+m))^{-\gamma},
$$

where we may choose $\gamma$ such that $\frac{1}{p_{1}}<\gamma<\frac{1}{p_{2}}$; this is possible since we are dealing with $p^{*}<\infty$, i.e., $p_{1}>p_{2}$. Then $f_{\gamma}$ does not belong to $F_{p_{2}, q_{2}}^{s_{2}}\left(\mathbb{R}^{n}\right)$ as $\left\|f_{\gamma} \mid F_{p_{2}, q_{2}}^{s_{2}}\left(\mathbb{R}^{n}\right)\right\|$ diverges for $\gamma p_{2}<1$. On the other hand, (3.85) reads for $\delta=\frac{\alpha_{1}}{p_{1}}, \frac{\alpha_{2}}{p_{1}}=\frac{1}{p^{*}}=\frac{1}{p_{2}}-\frac{1}{p_{1}}$ as

$$
\begin{aligned}
& \left\|f_{\gamma} \mid F_{p_{1}, q_{1}}^{s_{1}}\left(\mathbb{R}^{n}, w_{(\boldsymbol{\alpha}, \boldsymbol{\beta})}\right)\right\| \\
& \quad \leq c\left(\sum_{m=0}^{\infty} 2^{m\left(\delta-\frac{\alpha_{1}}{p_{1}}\right) p_{1}}(1+m)^{\left(\frac{\alpha_{2}}{p_{1}}-\frac{1}{p_{2}}\right) p_{1}}(1+\log (1+m))^{-\gamma p_{1}}\right)^{1 / p_{1}} \\
& \quad=c\left(\sum_{k=1}^{\infty} \frac{1}{k(1+\log k)^{\gamma p_{1}}}\right)^{1 / p_{1}}
\end{aligned}
$$


which converges by our choice of $\gamma$. Consequently we cannot have an embedding of type (3.77) in this situation.

Step 3. Conversely, we have to prove the compactness of (3.77) under the assumptions of (ii). We may assume again that we deal with the limiting situations $\delta=\frac{\alpha_{1}}{p_{1}}>\frac{n}{p^{*}}$ again. Assume first $\frac{\beta_{1}}{p_{1}}>\frac{n}{p^{*}}, \beta_{2} \in \mathbb{R}$ in (3.33). Since $s_{1}>s_{2}$ we may choose numbers $r_{i}$ and $\sigma_{i}, i=1,2$, such that $\frac{p_{1}}{p^{*}}<\frac{r_{1}}{r^{*}}<\min \left(\alpha_{2}, \frac{\alpha_{1}}{n}, \frac{\beta_{1}}{n}\right)$, and $s_{1}>\sigma_{1} \geq \sigma_{2}>s_{2}$, satisfying (3.81); then Proposition 3.1(iii) and Proposition 3.9(iii) lead to the compactness of (3.77) in view of

$$
F_{p_{1}, q_{1}}^{s_{1}}\left(\mathbb{R}^{n}, w_{(\boldsymbol{\alpha}, \boldsymbol{\beta})}\right) \hookrightarrow B_{r_{1}, p_{1}}^{\sigma_{1}}\left(\mathbb{R}^{n}, w_{(\boldsymbol{\alpha}, \boldsymbol{\beta})}\right) \hookrightarrow B_{r_{2}, p_{2}}^{\sigma_{2}}\left(\mathbb{R}^{n}\right) \hookrightarrow F_{p_{2}, q_{2}}^{s_{2}}\left(\mathbb{R}^{n}\right) .
$$

We turn to the case $\frac{\beta_{1}}{p_{1}}=\frac{n}{p^{*}}, \frac{\beta_{2}}{p_{1}}>\frac{1}{p^{*}}$, and begin with $p_{1}<p_{2}$, that is, $p^{*}=\infty, \beta_{1}=0, \beta_{2}>0, \alpha_{2}>0, \delta=\frac{\alpha_{1}}{p_{1}}>0$, in particular, $s_{1}>s_{2}$. Again we choose $\sigma_{i}$ and $r_{i}, i=1,2$, such that $s_{1}>\sigma_{1}>\sigma_{2}>s_{2}$, $p_{1}<r_{1}<r_{2}<p_{2}$, satisfy (3.81) with (3.82). Then we have the following chain of embeddings,

$$
\begin{aligned}
& F_{p_{1}, q_{1}}^{s_{1}}\left(\mathbb{R}^{n}, w_{(\boldsymbol{\alpha}, \boldsymbol{\beta})}\right) \stackrel{\mathrm{id}_{1}}{\longrightarrow} F_{p_{1}, \infty}^{s_{1}}\left(\mathbb{R}^{n}, w_{(\boldsymbol{\alpha}, \boldsymbol{\beta})}\right) \stackrel{\mathrm{id}_{2}}{\longrightarrow} F_{r_{1}, r_{1}}^{\sigma_{1}}\left(\mathbb{R}^{n}, w_{(\boldsymbol{\alpha}, \boldsymbol{\beta})}\right) \\
& \stackrel{\mathrm{id}_{3}}{\longrightarrow} F_{r_{2}, r_{2}}^{\sigma_{2}}\left(\mathbb{R}^{n}\right) \stackrel{\mathrm{id}_{4}}{\longrightarrow} F_{p_{2}, q_{2}}^{s_{2}}\left(\mathbb{R}^{n}\right),
\end{aligned}
$$

where $\mathrm{id}_{1}$ is continuous by (3.1), and $\mathrm{id}_{2}, \mathrm{id}_{4}$ are continuous due to (3.6) with $w=w_{(\boldsymbol{\alpha}, \boldsymbol{\beta})}, d=n+\alpha_{1}$, and $w \equiv 1, d=n$, respectively. Since $F_{r, r}^{\sigma}=B_{r, r}^{\sigma}, \mathrm{id}_{3}$ is compact in view of Proposition 3.9(iii) with $r^{*}=\infty, \beta_{1}=0, \beta_{2}>0, \alpha_{2}>0$ and $\widetilde{\delta}=\frac{\alpha_{1}}{r_{1}}$ by (3.82). Thus (3.77) is compact and it remains to deal with $p_{1} \geq p_{2}$. Clearly, Proposition 3.9(iii) together with (3.2) establishes the compactness of (3.77) when

$$
\frac{\alpha_{2}}{p_{1}}>\left(\frac{1}{\min \left(p_{2}, q_{2}\right)}-\frac{1}{\max \left(p_{1}, q_{1}\right)}\right) \geq \frac{1}{p^{*}} .
$$

In order to complete the proof of (3.80) we show that for $\delta=\frac{\alpha_{1}}{p_{1}}>\frac{n}{p^{*}}$ and $\frac{\alpha_{2}}{p_{1}}>\frac{\delta}{n}$ we also obtain compactness of (3.77). We apply some splitting argument together with (3.76). Let $\psi \in \mathcal{S}\left(\mathbb{R}^{n}\right)$ be compactly supported near the origin, $\operatorname{supp} \psi \subset\left\{x \in \mathbb{R}^{n}:|x|<3\right\}=\Omega$ with $\psi(x)=1$ for $|x| \leq 2$. By $\widetilde{F}_{p, q}^{s}(\Omega, w)$ we mean the subspace of $F_{p, q}^{s}\left(\mathbb{R}^{n}, w\right)$,

$$
\widetilde{F}_{p, q}^{s}(\Omega, w)=\left\{f \in F_{p, q}^{s}\left(\mathbb{R}^{n}, w\right): \operatorname{supp} f \subset \Omega\right\},
$$


as usual. We decompose

$$
f=\psi f+(1-\psi) f
$$

and consider the maps

$$
J_{\psi}: F_{p_{1}, q_{1}}^{s_{1}}\left(\mathbb{R}^{n}, w_{(\boldsymbol{\alpha}, \boldsymbol{\beta})}\right) \rightarrow \widetilde{F}_{p_{1}, q_{1}}^{s_{1}}\left(\Omega, w_{(\boldsymbol{\alpha}, \boldsymbol{\alpha})}\right), \quad f \mapsto \psi f,
$$

and

$$
J^{\psi}: F_{p_{1}, q_{1}}^{s_{1}}\left(\mathbb{R}^{n}, w_{(\boldsymbol{\alpha}, \boldsymbol{\beta})}\right) \rightarrow F_{p_{1}, q_{1}}^{s_{1}}\left(\mathbb{R}^{n}, w_{(\mathbf{0}, \boldsymbol{\beta})}\right), \quad f \mapsto(1-\psi) f .
$$

The idea is to prove that both, $J_{\psi}$ and $J^{\psi}$ are well-defined and can be appropriately extended to compact maps into $F_{p_{2}, q_{2}}^{s_{2}}\left(\mathbb{R}^{n}\right)$ such that $\operatorname{id}_{F}=I_{1}+I_{2}$ inherits their compactness finally. We begin with $J^{\psi}$. The well-definedness of it is immediate from the atomic decomposition characterisation of $F_{p, q}^{s}\left(\mathbb{R}^{n}, w\right), w \in \mathcal{A}_{\infty}$, in [24], since $\operatorname{supp}(1-\psi) f \cap B(0,2)=\emptyset$ such that there is no influence of the local part of $w_{(\boldsymbol{\alpha}, \boldsymbol{\beta})}$. Furthermore, since we have already proved the compactness of $\mathrm{id}_{F}$ in case of (3.79), this leads to the compactness of

$$
I_{2}: F_{p_{1}, q_{1}}^{s_{1}}\left(\mathbb{R}^{n}, w_{(\boldsymbol{\alpha}, \boldsymbol{\beta})}\right) \stackrel{J^{\psi}}{\longrightarrow} F_{p_{1}, q_{1}}^{s_{1}}\left(\mathbb{R}^{n}, w_{(\mathbf{0}, \boldsymbol{\beta})}\right) \stackrel{\mathrm{id}_{F}^{\prime}}{\longrightarrow} F_{p_{2}, q_{2}}^{s_{2}}\left(\mathbb{R}^{n}\right) .
$$

We deal with $J_{\psi}$. Again the atomic decomposition ensures that

$$
\left\|\psi f\left|\widetilde{F}_{p_{1}, q_{1}}^{s_{1}}\left(\Omega, w_{(\boldsymbol{\alpha}, \boldsymbol{\beta})}\right)\|\sim\| \psi f\right| \widetilde{F}_{p_{1}, q_{1}}^{s_{1}}\left(\Omega, w_{(\boldsymbol{\alpha}, \boldsymbol{\alpha})}\right)\right\|
$$

and $J_{\psi}$ is well-defined (in fact, we could have chosen any $\gamma$ for the target space $\widetilde{F}_{p_{1}, q_{1}}^{s_{1}}\left(\Omega, w_{(\boldsymbol{\alpha}, \gamma)}\right)$, but have taken $\boldsymbol{\alpha}=\boldsymbol{\gamma}$ for convenience, as will be clear soon). Now we refine this decomposition further and study

$$
\begin{aligned}
& I_{1}: F_{p_{1}, q_{1}}^{s_{1}}\left(\mathbb{R}^{n}, w_{(\boldsymbol{\alpha}, \boldsymbol{\beta})}\right) \stackrel{J_{\psi}}{\longrightarrow} \widetilde{F}_{p_{1}, q_{1}}^{s_{1}}\left(\Omega, w_{(\boldsymbol{\alpha}, \boldsymbol{\alpha})}\right) \stackrel{\text { re }}{\longrightarrow} F_{p_{1}, q_{1}}^{s_{1}}\left(\Omega, w_{(\boldsymbol{\alpha}, \boldsymbol{\alpha})}\right) \\
& \stackrel{\mathrm{id}_{\Omega}}{\longrightarrow} F_{p_{2}, q_{2}}^{s_{2}}(\Omega) \stackrel{\text { ext }}{\longrightarrow} F_{p_{2}, q_{2}}^{s_{2}}\left(\mathbb{R}^{n}\right),
\end{aligned}
$$

where re and ext are the usual (bounded) restriction and extension (by zero) operators. Thus it is sufficient to show the compactness of $\mathrm{id}_{\Omega}$. By an (obviously) adapted local version of (3.68) (with $\alpha_{i}=\beta_{i}>0, i=1,2$, cf. Remark 3.13) we know that

$$
L_{p_{1}}\left(\Omega, w_{(\boldsymbol{\alpha}, \boldsymbol{\alpha})}\right) \hookrightarrow L_{r, p_{1}}(\log L)_{\alpha_{2} / p_{1}}(\Omega), \quad \frac{1}{r}=\frac{1}{p_{1}}+\frac{\alpha_{1}}{n p_{1}},
$$


and, by the definition of Lorentz-Zygmund spaces, see [1, Thm. 9.3], that

$$
L_{r, p_{1}}(\log L)_{\alpha_{2} / p_{1}}(\Omega) \hookrightarrow L_{r}(\log L)_{\theta}(\Omega) \quad \text { for } \quad \theta<\frac{\alpha_{2}}{p_{1}}-\frac{\alpha_{1}}{n p_{1}},
$$

since $r<p_{1}$. Thus, by construction,

$$
F_{p_{1}, q_{1}}^{s_{1}}\left(\Omega, w_{(\boldsymbol{\alpha}, \boldsymbol{\alpha})}\right) \hookrightarrow F_{q_{1}}^{s_{1}}\left(L_{r}(\log L)_{\theta}\right)(\Omega), \quad \frac{1}{r}=\frac{1}{p_{1}}+\frac{\alpha_{1}}{n p_{1}}, \quad \theta<\frac{\alpha_{2}}{p_{1}}-\frac{\alpha_{1}}{n p_{1}} .
$$

Now we apply (3.76) with $a_{1}=\theta>0=a_{2}$ and obtain compactness of

$$
\operatorname{id}_{\Omega}: F_{p_{1}, q_{1}}^{s_{1}}\left(\Omega, w_{(\boldsymbol{\alpha}, \boldsymbol{\alpha})}\right) \hookrightarrow F_{q_{1}}^{s_{1}}\left(L_{r, r}(\log L)_{\theta}\right)(\Omega) \stackrel{\mathrm{id}_{F, \log }^{\Omega}}{\longrightarrow} F_{p_{2}, q_{2}}^{s_{2}}(\Omega)
$$

since $\delta=\frac{\alpha_{1}}{p_{1}}$ implies the required limiting condition $s_{1}-\frac{n}{r}=$ $s_{1}-\frac{n}{p_{1}}-\frac{\alpha_{1}}{p_{1}}=s_{2}-\frac{n}{p_{2}}$ in (3.75). This finally leads to the compactness of $I_{1}$, hence $\operatorname{id}_{F}$, if we can choose $\theta$ appropriately, that is, when $\frac{\alpha_{2}}{p_{1}}>\frac{\alpha_{1}}{n p_{1}}=\frac{\delta}{n}$.

Remark 3.16. It is obvious that in the above result the sufficiency (ii) of the compactness of $\operatorname{id}_{F}$ given by (3.77) and the necessity (i) only differ by the lower bound of $\alpha_{2}$ in the limiting situation when $\delta=\frac{\alpha_{1}}{p_{1}}>\frac{n}{p^{*}}=\frac{\beta_{1}}{p_{1}}, \frac{\beta_{2}}{p_{1}}>\frac{1}{p^{*}}$, and, in addition, $q_{1}>p_{1} \geq p_{2}$ or $p_{1} \geq p_{2}>q_{2}$. We believe that (i) represents the appropriate criterion for the compactness of (3.77), i.e., that $\mathrm{id}_{F}$ is compact if, and only if, conditions (3.33) and (3.78) are satisfied. But this stronger version of (ii) is not yet covered by our above proof.

Remark 3.17. In [25] we studied compact embeddings for weights of type (3.15) and obtained the criterion recalled in Proposition 3.6(iii), whereas in [26] we paid attention to general approaches for $w \in \mathcal{A}_{\infty}$. In [26, Cor. 3.5] we proved that for

$$
\delta>\frac{n}{p^{*}}+\frac{n}{p_{1}}\left(r_{w}-1\right)
$$

the embedding $\operatorname{id}_{w}: A_{p_{1}, q_{1}}^{s_{1}}\left(\mathbb{R}^{n}, w\right) \rightarrow A_{p_{2}, q_{2}}^{s_{2}}\left(\mathbb{R}^{n}\right)$ is compact if, and only if,

(3.88)

$$
\left\{w\left(Q_{0, m}\right)^{-1 / p_{1}}\right\}_{m \in \mathbb{Z}^{n}} \in \ell_{p^{*}} \text { with } \lim _{|m| \rightarrow \infty} w\left(Q_{0, m}\right)=\infty \quad \text { if } \quad p^{*}=\infty .
$$

In case of $w=w_{(\boldsymbol{\alpha}, \boldsymbol{\beta})}$ the characteristic number $r_{w}$ is given by $r_{w_{(\boldsymbol{\alpha}, \boldsymbol{\beta})}}=1+\frac{\max \left(\alpha_{1}, \beta_{1}, 0\right)}{n}$. Moreover, in view of (3.35) condition (3.88) 
corresponds to (3.33), whereas (3.87), reading in this particular case as $\delta>\frac{n}{p^{*}}+\frac{\max \left(\alpha_{1}, 0\right)}{p_{1}}$ only partially covers (3.34). We discussed this phenomenon in [26] in further detail.

\section{Entropy numbers of compact embeddings}

We finally study the 'degree' of compactness of $\operatorname{id}_{B}$ given by (3.27) in detail, using the well-known concept of entropy numbers which we shall recall first.

Let $X, Y$ be two quasi-Banach spaces and let $T: X \rightarrow Y$ be a bounded linear operator. The $k$-th (dyadic) entropy number of $T$, $k \in \mathbb{N}$, is defined as

$e_{k}(T)=\inf \left\{\varepsilon>0: T\left(B_{X}\right)\right.$ can be covered by $2^{k-1}$ balls of radius $\varepsilon$ in $\left.Y\right\}$,

where $B_{X}$ denotes the closed unit ball in $X$. Due to the well known fact that

$T: X \rightarrow Y$ is compact if, and only if, $\quad \lim _{k \rightarrow \infty} e_{k}(T: X \rightarrow Y)=0$,

the entropy numbers can be viewed as a quantification of the notion of compactness. Further properties like multiplicativity and additivity, as well as applications can be found in $[9,11,13,38]$.

We begin with our main result in [25] for weights of type (3.15) which can be seen as special cases of $w_{(\boldsymbol{\alpha}, \boldsymbol{\beta})}$ with $\alpha_{2}=\beta_{2}=0$.

Theorem 4.1. Let the parameters satisfy (3.14) and let the weight $w_{\alpha_{1}, \beta_{1}} \in \mathcal{A}_{\infty}$ be of type (3.15) with

$$
\frac{\beta_{1}}{p_{1}}>\frac{n}{p *}, \quad \alpha_{1}>-n \quad \text { and } \delta>\max \left(\frac{n}{p *}, \frac{\alpha_{1}}{p_{1}}\right) .
$$

(i) If $\frac{\beta_{1}}{p_{1}} \neq \delta$, then

$$
e_{k}\left(A_{p_{1}, q_{1}}^{s_{1}}\left(\mathbb{R}^{n}, w_{\alpha_{1}, \beta_{1}}\right) \hookrightarrow A_{p_{2}, q_{2}}^{s_{2}}\left(\mathbb{R}^{n}\right)\right) \sim k^{-\min \left(\frac{\beta_{1}}{n p_{1}}+\frac{1}{p_{1}}-\frac{1}{p_{2}}, \frac{s_{1}-s_{2}}{n}\right)} .
$$

(ii) If $\frac{\beta_{1}}{p_{1}}=\delta$ and $\tau=\frac{s_{1}-s_{2}}{n}+\frac{1}{q_{2}}-\frac{1}{q_{1}} \neq 0$, then

$$
e_{k}\left(B_{p_{1}, q_{1}}^{s_{1}}\left(\mathbb{R}^{n}, w_{\alpha_{1}, \beta_{1}}\right) \hookrightarrow B_{p_{2}, q_{2}}^{s_{2}}\left(\mathbb{R}^{n}\right)\right) \sim k^{-\frac{s_{1}-s_{2}}{n}}(1+\log k)^{\tau_{+}} .
$$


Remark 4.2. There is a parallel result for the related doubleweighted situation in [25, Thm. 3.6].

We now study compact embeddings of spaces with weights of type (3.21) with (3.22). It follows from Theorem 2.8 that the investigation of the asymptotic behaviour of entropy numbers of the embedding

$$
B_{p_{1}, q_{1}}^{s_{1}}\left(\mathbb{R}^{n}, w\right) \hookrightarrow B_{p_{2}, q_{2}}^{s_{2}}\left(\mathbb{R}^{n}\right)
$$

can be reduced to the estimation of the asymptotic behaviour of entropy numbers of embeddings of corresponding sequence spaces $b_{p_{1}, q_{1}}^{s_{1}}(w) \hookrightarrow b_{p_{2}, q_{2}}^{s_{2}}$. To make the notation more transparent we introduce the following spaces,

$$
\ell_{q}\left(2^{j \theta} \ell_{p}\left(\mathbb{Z}^{n}, w\right)\right)=\left\{\lambda=\left\{\lambda_{j, m}\right\}_{j, m}: \quad \lambda_{j, m} \in \mathbb{C}, j \in \mathbb{N}_{0}, m \in \mathbb{Z}^{n},\right.
$$

$$
\left.\left\|\lambda \mid \ell_{q}\left(2^{j \theta} \ell_{p}\left(\mathbb{Z}^{n}, w\right)\right)\right\|=\left(\sum_{j=0}^{\infty} 2^{j \theta q}\left(\sum_{m \in \mathbb{Z}^{n}}\left|\lambda_{j, m}\right|^{p} w(j, m)\right)^{\frac{q}{p}}\right)^{\frac{1}{q}}<\infty\right\},
$$

$\ell_{q}\left(2^{j \theta} \ell_{p}^{M_{j}}\left(\mathbb{Z}^{n}, w\right)\right)=\left\{\left\{s_{j, m}\right\}_{j, m} \in \ell_{q}\left(2^{j \theta} \ell_{p}\left(\mathbb{Z}^{n}, w\right)\right): s_{j, m}=0 \quad\right.$ if $\left.\quad|m|>M_{j}\right\}$,

$\ell_{q}\left(2^{j \theta} \tilde{\ell}_{p}^{M_{j}}\left(\mathbb{Z}^{n}, w\right)\right)=\left\{\left\{s_{j, m}\right\}_{j, m} \in \ell_{q}\left(2^{j \theta} \ell_{p}\left(\mathbb{Z}^{n}, w\right)\right): s_{j, m}=0 \quad\right.$ if $\left.\quad|m| \leq M_{j}\right\}$,

(with the usual modification in (4.3) when $p=\infty$ and/or $q=\infty$ ), $M_{j} \in \mathbb{N}$. In an analogous way we define the space $\ell_{q}\left(2^{j \theta} \ell_{p}(\mathbb{N}, w)\right)$ and its subspaces. Our plan is to adapt arguments used in the proof of Theorem 4.1 in [25] as well as to apply Theorem 4.1 directly based on Corollary 3.11. For this purpose we recall [25, Lemma 3.1] adapted to our present notation.

Lemma 4.3. Let $0<p_{1}<\infty, 0<p_{2} \leq \infty, 0<q_{1}, q_{2} \leq \infty, \xi \in \mathbb{R}$, $\theta>0$ and $M \in \mathbb{N}$. Let $w_{\xi}(j, m)=|m|^{\xi}$ if $m \neq 0$ and $w_{\xi}(j, 0)=1$, $j \in \mathbb{N}_{0}$. Assume $\frac{\xi}{p_{1}}>-\theta+\frac{n}{p^{*}}$. Then there are positive constants $c$ and $C$ such that for all $k \in \mathbb{N}$ the estimates

$$
\begin{aligned}
c k^{-\left(\frac{\theta}{n}+\frac{\xi}{n p_{1}}+\frac{1}{p_{1}}-\frac{1}{p_{2}}\right)} & \leq e_{k}\left(\mathrm{id}: \ell_{q_{1}}\left(2^{j \theta} \ell_{p_{1}}^{M 2^{j}}\left(\mathbb{Z}^{n}, w_{\xi}\right)\right) \rightarrow \ell_{q_{2}}\left(\ell_{p_{2}}^{M 2^{j}}\left(\mathbb{Z}^{n}\right)\right)\right) \\
& \leq C k^{-\left(\frac{\theta}{n}+\frac{\xi}{n p_{1}}+\frac{1}{p_{1}}-\frac{1}{p_{2}}\right)}
\end{aligned}
$$

hold. 
The next tool we need is the following extended version of [32, Cors. 4.18, 4.19] and [33, Thm. 1, Prop. 4]. Let the weight $w^{\beta_{1}, \beta_{2}}$, $\beta_{1} \geq 0, \beta_{2} \in \mathbb{R}$, be given by

(4.6) $w^{\beta_{1}, \beta_{2}}(j, m)=\left(1+2^{-j}|m|\right)^{\beta_{1}}\left(1+\log \left(1+2^{-j}|m|\right)\right)^{\beta_{2}}, \quad j \in \mathbb{N}_{0}, m \in \mathbb{Z}^{n}$,

which corresponds to some weight $\widetilde{w}_{(\mathbf{0}, \boldsymbol{\beta})}(x)$ given by (3.21) with $\boldsymbol{\alpha}=\mathbf{0}=(0,0), \boldsymbol{\beta}=\left(\beta_{1}, \beta_{2}\right)$, and $w^{\beta_{1}, \beta_{2}}(j, m) \sim \widetilde{w}_{(\mathbf{0}, \boldsymbol{\beta})}\left(2^{-j} m\right), j \in \mathbb{N}_{0}$, $m \in \mathbb{Z}^{n}$.

Proposition 4.4. Let the parameters satisfy (3.14) with $\delta>0$, recall (3.12).

(i) Assume $\beta_{1}>0, \beta_{2} \in \mathbb{R}$. Then the embedding

$$
\operatorname{id}^{\beta_{1}, \beta_{2}}: \ell_{q_{1}}\left(2^{j \delta} \ell_{p_{1}}\left(w^{\beta_{1}, \beta_{2}}\right)\right) \hookrightarrow \ell_{q_{2}}\left(\ell_{p_{2}}\right)
$$

is compact if, and only if,

$$
\text { either } \min \left(\delta, \frac{\beta_{1}}{p_{1}}\right)>\frac{n}{p^{*}}, \beta_{2} \in \mathbb{R}, \quad \text { or } \quad \delta>\frac{\beta_{1}}{p_{1}}=\frac{n}{p^{*}}, \frac{\beta_{2}}{p_{1}}>\frac{1}{p^{*}} \text {. }
$$

(ii) In case of $\min \left(\delta, \frac{\beta_{1}}{p_{1}}\right)>\frac{n}{p^{*}}, \beta_{2} \in \mathbb{R}$, then for all $k \in \mathbb{N}$,

$$
e_{k}\left(\mathrm{id}^{\beta_{1}, \beta_{2}}\right) \sim \begin{cases}k^{-\frac{\beta_{1}}{n p_{1}}-\frac{1}{p_{1}}+\frac{1}{p_{2}}}(1+\log k)^{-\frac{\beta_{2}}{p_{1}}}, & \text { if } \frac{n}{p^{*}}<\frac{\beta_{1}}{p_{1}}<\delta, \\ k^{-\frac{s_{1}-s_{2}}{n}}, & \text { if } \frac{n}{p^{*}}<\delta<\frac{\beta_{1}}{p_{1}}\end{cases}
$$

whereas for $\frac{n}{p^{*}}<\delta=\frac{\beta_{1}}{p_{1}}$ we obtain in case of $\tau=\frac{s_{1}-s_{2}}{n}+\frac{1}{q_{2}}-$ $\frac{1}{q_{1}} \neq \max \left(0, \frac{\beta_{2}}{p_{1}}\right)$,

$$
e_{k}\left(\operatorname{id}^{\beta_{1}, \beta_{2}}\right) \sim \begin{cases}k^{-\frac{s_{1}-s_{2}}{n}}, & \text { if } \frac{\beta_{2}}{p_{1}}>\max (\tau, 0), \\ k^{-\frac{s_{1}-s_{2}}{n}}(1+\log k)^{\tau-\frac{\beta_{2}}{p_{1}}}, & \text { if } \frac{\beta_{2}}{p_{1}} \leq 0<\tau, \\ & \text { or } 0<\frac{\beta_{2}}{p_{1}}<\tau \leq \frac{\beta_{2}}{p_{1}}+\frac{1}{q_{2}}-\frac{1}{q_{1}}, \\ k^{-\frac{s_{1}-s_{2}}{n}}(1+\log k)^{-\frac{\beta_{2}}{p_{1}}}, & \text { if } \beta_{2} \leq 0, \tau<0 .\end{cases}
$$


(iii) In case of $\delta>\frac{\beta_{1}}{p_{1}}=\frac{n}{p^{*}}, \frac{\beta_{2}}{p_{1}}>\frac{1}{p^{*}}$, then for $p^{*}<\infty$ we obtain for all $k \in \mathbb{N}$,

$$
\begin{aligned}
& \quad e_{k}\left(\operatorname{id}^{\beta_{1}, \beta_{2}}\right) \sim(1+\log k)^{-\frac{\beta_{2}}{p_{1}}+\frac{1}{p^{*}}}, \\
& \quad \text { whereas for } p^{*}=\infty \text {, that is, } \beta_{1}=0, \beta_{2}>0,
\end{aligned}
$$

$$
e_{k}\left(\mathrm{id}^{0, \beta_{2}}\right) \sim \begin{cases}k^{-\frac{\beta_{2}}{p_{1}}}, & \text { if } 0<\frac{\beta_{2}}{p_{1}} \leq \frac{1}{p_{1}}-\frac{1}{p_{2}}, \\ k^{-\frac{1}{p_{1}}+\frac{1}{p_{2}}}(1+\log k)^{-\frac{\beta_{2}}{p_{1}}+\frac{1}{p_{1}}-\frac{1}{p_{2}}}, & \text { if } \frac{\beta_{2}}{p_{1}}>\frac{1}{p_{1}}-\frac{1}{p_{2}} \geq 0 .\end{cases}
$$

Proof. Step 1. Part (i) coincides with [32, Cor. 5.8], and (4.9) is covered by [32, Cors. 4.18, 4.19]. The equivalence (4.11) was just recently proved in [29, Thm. 7], and [33, Thm. 1, Prop. 4] gives (4.12). Moreover, the lower estimates in (4.10) were shown in [30, Prop. 2], and the case $\beta_{2}=0$ coincides with [31, Thm. 2]. Hence it remains to deal with the corresponding upper estimates in (4.10).

Step 2. We always assume $\frac{n}{p^{*}}<\delta=\frac{\beta_{1}}{p_{1}}$ now; therefore

$$
\tau=\frac{\beta_{1}}{n p_{1}}+\frac{1}{p_{1}}-\frac{1}{p_{2}}+\frac{1}{q_{2}}-\frac{1}{q_{1}} .
$$

Recall that by [31, Thm. 2],

$$
e_{k}\left(\mathrm{id}^{\beta_{1}, 0}\right) \sim k^{-\frac{s_{1}-s_{2}}{n}}, \quad k \in \mathbb{N},
$$

if $\beta_{2}=0$ and $\tau<0$. Obviously, this implies by monotonicity

$$
e_{k}\left(\mathrm{id}^{\beta_{1}, \beta_{2}}\right) \leq c e_{k}\left(\mathrm{id}^{\beta_{1}, 0}\right)
$$

and thus the result for $\beta_{2} \geq 0>\tau$ in the first line in (4.10). As for the other cases in (4.10) we modify the ideas of the proof of [31, Thm. 2]. So we sketch here the proof and point out appropriate modifications.

Step 3. We divide $\mathrm{id}^{\beta_{1}, \beta_{2}}$ into two parts $\mathrm{id}^{\beta_{1}, \beta_{2}}=\mathrm{id}_{1}^{\beta_{1}, \beta_{2}}+\mathrm{id}_{2}^{\beta_{1}, \beta_{2}}$ where

$$
\left(\operatorname{id}_{1}^{\beta_{1}, \beta_{2}}(\lambda)\right)_{j, m}=\left\{\begin{array}{ll}
\lambda_{j, m}, & \text { if }|m| \leq 2^{j}, \\
0, & \text { if }|m|>2^{j},
\end{array} \quad \text { and } \quad \operatorname{id}_{2}^{\beta_{1}, \beta_{2}}=\mathrm{id}^{\beta_{1}, \beta_{2}}-\mathrm{id}_{1}^{\beta_{1}, \beta_{2}}\right.
$$


For the first part we have

$$
e_{k}\left(\operatorname{id}_{1}^{\beta_{1}, \beta_{2}}\right) \sim e_{k}\left(\operatorname{id}: \ell_{q_{1}}\left(2^{j \delta} \ell_{p_{1}}^{2^{j}}\left(\mathbb{Z}^{n}\right)\right) \rightarrow \ell_{q_{2}}\left(\ell_{p_{2}}^{2^{j}}\left(\mathbb{Z}^{n}\right)\right)\right) \sim k^{-\frac{s_{1}-s_{2}}{n}},
$$

cf. [48, Thm. 8.2]. On the other hand, similar to [31, Lemma 2]

$$
\begin{aligned}
e_{k}\left(\operatorname{id}_{2}^{\beta_{1}, \beta_{2}}\right) & \sim e_{k}\left(\mathrm{id}: \ell_{q_{1}}\left(\tilde{\ell}_{p_{1}}^{\ell^{j}}\left(\mathbb{Z}^{n}, w^{\beta_{1}, \beta_{2}}\right)\right) \rightarrow \ell_{q_{2}}\left(\tilde{\ell}_{p_{2}}^{2^{j}}\left(\mathbb{Z}^{n}\right)\right)\right) \\
& \sim e_{k}\left(D_{\beta_{1}, \beta_{2}}: \ell_{q_{1}}\left(\tilde{\ell}_{p_{1}}^{n^{n j}}(\mathbb{N})\right) \rightarrow \ell_{q_{2}}\left(\tilde{\ell}_{p_{2}}^{2^{n j}}(\mathbb{N})\right)\right),
\end{aligned}
$$

where $D_{\beta_{1}, \beta_{2}}$ is a diagonal operator defined by

$$
\left(D_{\beta_{1}, \beta_{2}} \lambda\right)_{j, k}=k^{-\frac{\beta_{1}}{n p_{1}}}\left(1+\log \left(1+2^{-j} k^{1 / n}\right)\right)^{-\frac{\beta_{2}}{p_{1}}} \lambda_{j, k}, \quad j \in \mathbb{N}_{0}, k>2^{n j} .
$$

We consider the projections $P_{N}, N \in \mathbb{N}$,

$$
\left(P_{N} \lambda\right)_{j, k}:= \begin{cases}\lambda_{j, k} & \text { if } 0 \leq j<N \quad \text { and } \quad k \in \mathbb{N}, \\ 0 & \text { if } j \geq N\end{cases}
$$

and decompose $D_{\beta_{1}, \beta_{2}}$ as

$$
D_{\beta_{1}, \beta_{2}}=D_{\beta_{1}, \beta_{2}} P_{N}+D_{\beta_{1}, \beta_{2}}\left(\mathrm{Id}-P_{N}\right),
$$

where Id denotes the identity. Applying Hölder's inequality, if necessary, yields

$$
\text { 18) }\left\|D_{\beta_{1}, \beta_{2}}\left(\operatorname{Id}-P_{N}\right) \mid \ell_{q_{1}}\left(\tilde{\ell}_{p_{1}}^{2^{n j}}(\mathbb{N})\right) \rightarrow \ell_{q_{2}}\left(\tilde{\ell}_{p_{2}}^{2 j j}(\mathbb{N})\right)\right\| \leq c_{1} 2^{-N\left(\frac{\beta_{1}}{p_{1}}-\frac{n}{p^{*}}\right)}
$$

for some constant $c_{1}$ independent of $N$.

Step 4. Let $\beta_{2}<0$. In order to estimate the entropy numbers of the operator $D_{\beta_{1}, \beta_{2}} P_{N}$ we consider the diagonal operator

$$
\left(D_{\beta_{1}, \beta_{2}}^{(j)} \lambda\right)_{k}:=k^{-\frac{\beta_{1}}{n p_{1}}}\left(1+\log \left(1+2^{-j} k^{\frac{1}{n}}\right)\right)^{-\frac{\beta_{2}}{p_{1}}} \lambda_{k}, \lambda=\left\{\lambda_{k}\right\} \in \ell_{p_{1}}, j=0,1, \ldots, N,
$$

and its vector-valued counterparts

$$
\widetilde{D} \lambda:=\left(D_{\beta_{1}, \beta_{2}}^{(0)} \lambda_{1}, \ldots, D_{\beta_{1}, \beta_{2}}^{(0)} \lambda_{N}\right), \quad \lambda=\left(\lambda_{1}, \ldots, \lambda_{N}\right) \in \ell_{q_{1}}^{N}\left(\ell_{p_{1}}\right),
$$


$(4.21)$

$\widehat{D} \lambda:=\left(D_{-\beta_{1},-\beta_{2}}^{(0)} D_{\beta_{1}, \beta_{2}}^{(0)} \lambda_{1}, \ldots, D_{-\beta_{1},-\beta_{2}}^{(0)} D_{\beta_{1}, \beta_{2}}^{(N-1)} \lambda_{N}\right), \quad \lambda=\left(\lambda_{1}, \ldots, \lambda_{N}\right) \in \ell_{q_{1}}^{N}\left(\ell_{p_{1}}\right)$.

Then $\widehat{D}: \ell_{q_{1}}^{N}\left(\ell_{p_{1}}\right) \rightarrow \ell_{q_{1}}^{N}\left(\ell_{p_{1}}\right)$ is a bounded operator with the norm uniformly bounded and $D_{\beta_{1}, \beta_{2}} P_{N}=\widetilde{D} \widehat{D} P_{N}$. Thus

$$
e_{k}\left(D_{\beta_{1}, \beta_{2}} P_{N}\right) \leq C e_{k}(\widetilde{D}) .
$$

It was proved in [28, Thm. 2.2] that if $\frac{\beta_{1}}{n p_{1}}>\frac{1}{p^{*}}$, then

$$
e_{k}\left(D_{\beta_{1}, \beta_{2}}^{(0)}: \ell_{p_{1}} \rightarrow \ell_{p_{2}}\right) \sim k^{-\gamma}(1+\log (1+k))^{-\beta_{2} / p_{1}}
$$

where $\gamma=\frac{\beta_{1}}{n p_{1}}+\frac{1}{p_{1}}-\frac{1}{p_{2}}=\frac{s_{1}-s_{2}}{n}>0$; hence in view of (4.13)

$$
\tau=\gamma+\frac{1}{q_{2}}-\frac{1}{q_{1}}
$$

To simplify notation we write $X=\ell_{q_{1}}^{N}\left(\ell_{p_{1}}\right)$ and $Y=\ell_{q_{2}}^{N}\left(\ell_{p_{2}}\right)$. Let $B_{X}$ denote the unit ball in $X$. For arbitrary natural numbers $k_{1}, \ldots, k_{N}$ we set

$$
\varepsilon_{j}:=2 c_{2} j^{-1 / q_{1}} k_{j}^{-\gamma}\left(1+\log k_{j}\right)^{-\frac{\beta_{2}}{p_{1}}} \quad \text { and } \quad \varepsilon:=\left(\sum_{j=1}^{N} \varepsilon_{j}^{q_{2}}\right)^{1 / q_{2}} .
$$

One can construct an $\varepsilon$-net in $Y$ for $\widetilde{D}\left(B_{X}\right)$ of cardinality at most $2^{K}$, where

$$
K:=N \log N+\sum_{j=1}^{N} k_{j},
$$

cf. [31]. Finally we choose $k_{j}$ in an appropriate way. Fix $k \in \mathbb{N}$, (4.26)

$$
N:=\varrho k \quad \text { where } \varrho \text { is defined by the equation } \quad \varrho\left(\frac{\beta_{1}}{p_{1}}-\frac{n}{p^{*}}\right)=\gamma \text {. }
$$

Then (4.18) becomes

$$
\left\|D_{\beta_{1}, \beta_{2}}\left(\mathrm{Id}-P_{N}\right) \mid \ell_{q_{1}}\left(\tilde{\ell}_{p_{1}}^{2^{n j}}(\mathbb{N})\right) \rightarrow \ell_{q_{2}}\left(\tilde{\ell}_{p_{2}}^{2^{n j}}(\mathbb{N})\right)\right\| \leq c_{1} 2^{-\gamma k} .
$$

Now we distinguish two cases. 
Case 1. Let $\tau<0$, i.e., $\gamma<\frac{1}{q_{1}}-\frac{1}{q_{2}}$ in view of (4.24). We choose $\theta>1$ such that $\gamma \theta<\frac{1}{q_{1}}-\frac{1}{q_{2}}$ and set

$$
k_{j}:=\left[j^{-\theta} 2^{k}\right], \quad j=1, \ldots, N .
$$

Since $\theta>1$ we obtain $K \sim 2^{k}$ by (4.25) and $\varepsilon \leq c 2^{-\gamma k} k^{-\beta_{2} / p_{1}}$. We conclude, for $m=c 2^{k}$ and $\beta_{2}<0$ that

$$
\begin{aligned}
e_{m}\left(D_{\beta_{1}, \beta_{2}}\right) & \leq e_{m}\left(D_{\beta_{1}, \beta_{2}} P_{N}\right)+\left\|D_{\beta_{1}, \beta_{2}}\left(\operatorname{Id}-P_{N}\right) \mid \ell_{q_{1}}\left(\tilde{\ell}_{p_{1}}^{2^{n j}}(\mathbb{N})\right) \rightarrow \ell_{q_{2}}\left(\tilde{\ell}_{p_{2}}^{2 j}(\mathbb{N})\right)\right\| \\
& \leq c 2^{-\gamma k} k^{-\frac{\beta_{2}}{p_{1}}}+c 2^{-\gamma k} \\
& \leq c^{\prime} m^{-\gamma}(1+\log m)^{-\frac{\beta_{2}}{p_{1}}} .
\end{aligned}
$$

In view of (4.16) and (4.17) this proves the last line in (4.10), since $\gamma=\frac{s_{1}-s_{2}}{n}$ and $\beta_{2}<0$.

Case 2. Let $\tau>0$, i.e., $\gamma>\frac{1}{q_{1}}-\frac{1}{q_{2}}$ by (4.24). Select now $0<\theta<1$ such that $\gamma \theta>\frac{1}{q_{1}}-\frac{1}{q_{2}}$ and set $k_{j}$ as in the first case. Now we obtain $K \sim 2^{k} k^{1-\theta}$ and $\varepsilon \leq c 2^{-\gamma k} k^{-\beta_{2} / p_{1}+\tau-(1-\theta) \gamma}$ by (4.25). We conclude, for $m=c 2^{k} k^{1-\theta}$ and $\beta_{2}<0$ that

$$
\begin{aligned}
e_{m}\left(D_{\beta_{1}, \beta_{2}}\right) & \leq e_{m}\left(D_{\beta_{1}, \beta_{2}} P_{N}\right)+\left\|D_{\beta_{1}, \beta_{2}}\left(\operatorname{Id}-P_{N}\right) \mid \ell_{q_{1}}\left(\tilde{\ell}_{p_{1}}^{2^{n j}}(\mathbb{N})\right) \rightarrow \ell_{q_{2}}\left(\tilde{\ell}_{p_{2}}^{2 n j}(\mathbb{N})\right)\right\| \\
& \leq c 2^{-\gamma k} k^{-\frac{\beta_{2}}{p_{1}}+\tau-(1-\theta) \gamma}+c 2^{-\gamma k} \\
& \leq c^{\prime} m^{-\gamma}(1+\log m)^{\tau-\frac{\beta_{2}}{p_{1}}}+c^{\prime} m^{-\gamma}(1+\log m)^{\gamma(1-\theta)} \\
& \leq c^{\prime \prime} m^{-\gamma}(1+\log m)^{\tau-\frac{\beta_{2}}{p_{1}}}
\end{aligned}
$$

since $\beta_{2}<0$ and $\gamma(1-\theta)<\tau$, recall (4.24) and our choice of $\theta$. In the same way as above, using (4.16) and (4.17), we obtain part of the second line in (4.10), that is, when $\beta_{2}<0<\tau$.

Step 5. Let $\beta_{2}>0$. It remains to consider the case $\tau>0$, more precisely, we are left to prove

$$
e_{k}\left(\operatorname{id}^{\beta_{1}, \beta_{2}}\right) \leq c \begin{cases}k^{-\frac{s_{1}-s_{2}}{n}}, & \text { if } \frac{\beta_{2}}{p_{1}}>\tau>0, \\ k^{-\frac{s_{1}-s_{2}}{n}}(1+\log k)^{\tau-\frac{\beta_{2}}{p_{1}}}, & \text { if } 0<\frac{\beta_{2}}{p_{1}}<\tau \leq \frac{\beta_{2}}{p_{1}}+\frac{1}{q_{2}}-\frac{1}{q_{1}} .\end{cases}
$$

Using the same diagonal operators (4.19) we get

$$
e_{k}\left(D_{\beta_{1}, \beta_{2}}^{(j)}: \ell_{p_{1}} \rightarrow \ell_{p_{2}}\right) \sim k^{-\gamma}\left(1+\log \left(1+2^{-j} k^{\frac{1}{n}}\right)\right)^{-\beta_{2} / p_{1}},
$$


$\gamma=\frac{\beta_{1}}{n p_{1}}+\frac{1}{p_{1}}-\frac{1}{p_{2}}=\frac{s_{1}-s_{2}}{n}>0$, cf. [28, Thm. 2.2]. Moreover examining the proof of the above theorem in [28] one can observe easily that the constants in this equivalence are independent of $j$.

Let $B_{p}$ denote the unit ball in $\ell_{p}$. Let $\mathcal{N}_{j}$ be the $\varepsilon_{j}$-net for the set $D_{\beta_{1}, \beta_{2}}^{(j)}\left(B_{p_{1}}\right)$ in $\ell_{p_{2}}$ of cardinality $2^{k_{j}}$. If $0<r_{j} \leq 1$, then one can find an $\varepsilon_{j}$-net for the set $D_{\beta_{1}, \beta_{2}}^{(j)}\left(r_{j} B_{p_{1}}\right)$ in $\ell_{p_{2}}$ of the same cardinality $2^{k_{j}}$. If

$$
\varepsilon_{r}:=\left(\sum_{j=1}^{N}\left(r_{j} \varepsilon_{j}\right)^{q_{2}}\right)^{1 / q_{2}}, \quad r=\left(r_{1}, \ldots, r_{N}\right)
$$

then one can find the $\varepsilon$-net for

$$
D_{\beta_{1}, \beta_{2}}\left(r_{1} B_{p_{1}} \times r_{2} B_{p_{1}} \times \ldots \times r_{N} B_{p_{1}}\right)
$$

of cardinality $2^{k_{1}+\ldots+k_{N}}$ in $Y$.

In order to construct an $\varepsilon$-net in $Y$ for $D_{\beta_{1}, \beta_{2}}\left(B_{X}\right)$ we proceed as follows. For any permutation $\pi$ of the index set $\{1,2, \ldots, N\}$ we put

$B_{\pi}:=\left\{\lambda=\left(\lambda_{j}\right)_{j=1}^{N} \in B_{X}:\left\|\lambda_{\pi(1)}\left|\ell_{p_{1}}\|\geq\| \lambda_{\pi(2)}\right| \ell_{p_{1}}\right\| \geq \ldots \geq\left\|\lambda_{\pi(N)} \mid \ell_{p_{1}}\right\|\right\}$.

Then

$$
B_{X}=\bigcup_{\pi} B_{\pi}
$$

and $\lambda \in B_{\pi}$ implies $\left\|\lambda_{\pi(j)} \mid \ell_{p_{1}}\right\| \leq j^{-1 / q_{1}}$. Thus

$$
B_{\pi} \subset r_{1} B_{p_{1}} \times r_{2} B_{p_{1}} \times \ldots \times r_{N} B_{p_{1}} \quad \text { with } \quad r_{j}=\pi_{-1}(j)^{-1 / q_{1}}
$$

where $\pi_{-1}$ denotes the permutation inverse to $\pi$. We put

$$
k_{j}=(N-j+1)^{-\theta} 2^{k}, \quad \text { with } \theta>0, k \in \mathbb{N}, j=0,1, \ldots, N
$$

It follows from the estimates (4.29) that one can assume that

$$
\varepsilon_{1} \geq \varepsilon_{2} \geq \ldots \geq \varepsilon_{N}
$$

So if $r(\pi)=\left(\pi_{-1}(1)^{-1 / q_{1}}, \ldots, \pi_{-1}(N)^{-1 / q_{1}}\right)$, then

$$
\varepsilon_{r(\pi)}:=\left(\sum_{j=1}^{N}\left(\pi_{-1}(j)^{-1 / q_{1}} \varepsilon_{j}\right)^{q_{2}}\right)^{1 / q_{2}} \leq\left(\sum_{j=1}^{N} j^{-q_{2} / q_{1}} \varepsilon_{j}^{q_{2}}\right)^{1 / q_{2}}
$$




$$
\leq c\left(\sum_{j=1}^{N} j^{-q_{2} / q_{1}} k_{j}^{-q_{2} \gamma}\left(1+\log \left(1+2^{-j} k_{j}^{\frac{1}{n}}\right)\right)^{-\frac{q_{2} \beta_{2}}{p_{1}}}\right)^{1 / q_{2}}=: \varepsilon_{o} .
$$

In the same way as in Step 4 one can construct an $\varepsilon_{o}$-net in $\ell_{q_{2}}^{N}\left(\ell_{p_{2}}\right)$ for $D_{\beta_{1}, \beta_{2}}\left(B_{X}\right)$ of cardinality at most $2^{K}$, where $K$ is given by formula (4.25).

Let $k$ and $N$ be related by (4.26), then the definition of $k_{j}$ gives us $\left(1+\log \left(1+2^{-j} k_{j}^{\frac{1}{n}}\right)\right) \sim(N-j+1)$, and, consequently,

$$
\varepsilon_{o} \leq c 2^{-k \gamma}\left(\sum_{j=1}^{N} j^{-q_{2} / q_{1}}(N-j+1)^{q_{2} \theta \gamma-\frac{q_{2} \beta_{2}}{p_{1}}}\right)^{1 / q_{2}}
$$

If we assume $\theta \gamma-\frac{\beta_{2}}{p_{1}} \leq 0$, then the last inequality implies

$$
\varepsilon_{o} \leq c 2^{-k \gamma}\left(\sum_{j=1}^{N} j^{q_{2}\left(\theta \gamma-1 / q_{1}-\frac{\beta_{2}}{p_{1}}\right)}\right)^{1 / q_{2}} .
$$

Once more we distinguish two cases.

Case 1. Let $\tau-\frac{\beta_{2}}{p_{1}}<0$, i.e., $\gamma<\frac{\beta_{2}}{p_{1}}+\frac{1}{q_{1}}-\frac{1}{q_{2}}$ in view of (4.24).

If $q_{2} \leq q_{1}$, then we choose $\theta>1$ such that $\gamma \theta<\frac{\beta_{2}}{p_{1}}+\frac{1}{q_{1}}-\frac{1}{q_{2}}<\frac{\beta_{2}}{p_{1}}$. Since $\theta>1$ we obtain $K \sim 2^{k}$ by (4.25) and $\varepsilon_{o} \leq c 2^{-\gamma k}$. We conclude, for $m=c 2^{k}$ and $\gamma=\frac{s_{1}-s_{2}}{n} \leq \frac{\beta_{2}}{p_{1}}$ that

$$
\begin{aligned}
e_{m}\left(D_{\beta_{1}, \beta_{2}}\right) & \leq e_{m}\left(D_{\beta_{1}, \beta_{2}} P_{N}\right)+\left\|D_{\beta_{1}, \beta_{2}}\left(\mathrm{Id}-P_{N}\right) \mid \ell_{q_{1}}\left(\tilde{\ell}_{p_{1}}^{n j}(\mathbb{N})\right) \rightarrow \ell_{q_{2}}\left(\tilde{\ell}_{p_{2}}^{2^{n j}}(\mathbb{N})\right)\right\| \\
& \leq c 2^{-\gamma k} \leq c^{\prime} m^{-\gamma} .
\end{aligned}
$$

If $q_{2}>q_{1}$, then we choose $\widetilde{q_{1}}=\widetilde{q_{2}}=q_{2}$. Now the estimate follows from elementary embeddings of the sequence spaces and the above considerations. This finishes the proof of the first line in (4.10).

Case 2. It remains to deal with $0<\tau-\frac{\beta_{2}}{p_{1}} \leq \frac{1}{q_{2}}-\frac{1}{q_{1}}$. Select now $0<\theta<1$ such that $\gamma \theta>\frac{\beta_{2}}{p_{1}}+\frac{1}{q_{1}}-\frac{1}{q_{2}}$. We obtain $K \sim 2^{k} k^{1-\theta}$ and $\varepsilon_{o} \leq c 2^{-\gamma k} k^{\theta \gamma+1 / q_{2}-1 / q_{1}-\beta_{2} / p_{1}}$. Thus for $m=c 2^{k} k^{1-\theta}$ and $\gamma=\frac{s_{1}-s_{2}}{n} \leq \frac{\beta_{2}}{p_{1}}$ we conclude that

$$
\begin{aligned}
e_{m}\left(D_{\beta_{1}, \beta_{2}}\right) & \leq e_{m}\left(D_{\beta_{1}, \beta_{2}} P_{N}\right)+\left\|D_{\beta_{1}, \beta_{2}}\left(\operatorname{Id}-P_{N}\right) \mid \ell_{q_{1}}\left(\tilde{\ell}_{p_{1}}^{2^{n j}}(\mathbb{N})\right) \rightarrow \ell_{q_{2}}\left(\tilde{\ell}_{p_{2}}^{2^{n j}}(\mathbb{N})\right)\right\| \\
& \leq c 2^{-\gamma k} k^{\theta \gamma+1 / q_{2}-1 / q_{1}-\beta_{2} / p_{1}}+c 2^{-\gamma k} \leq c^{\prime \prime} m^{-\gamma}(1+\log m)^{\tau-\frac{\beta_{2}}{p_{1}}} .
\end{aligned}
$$


This finally completes the proof of (4.10).

Remark 4.5. The assumption concerning the upper bounds for $\tau$ of the form $\tau<\frac{\beta_{2}}{p_{1}}+\frac{1}{q_{2}}-\frac{1}{q_{1}}$ in (4.10) is not natural and it is expected not to be necessary. We conjecture that the estimate holds without this restriction, that is,

$$
\begin{aligned}
e_{k}\left(\mathrm{id}^{\beta_{1}, \beta_{2}}\right) \sim k^{-\frac{s_{1}-s_{2}}{n}}(1+\log k)^{\left(\tau+-\frac{\beta_{2}}{p_{1}}\right)_{+}} \\
\qquad \begin{array}{ll}
k^{-\frac{s_{1}-s_{2}}{n}}, & \text { if } \frac{\beta_{2}}{p_{1}}>\max (\tau, 0), \\
k^{-\frac{s_{1}-s_{2}}{n}}(1+\log k)^{\tau-\frac{\beta_{2}}{p_{1}}}, & \text { if } \tau>\max \left(\frac{\beta_{2}}{p_{1}}, 0\right), \\
k^{-\frac{s_{1}-s_{2}}{n}}(1+\log k)^{-\frac{\beta_{2}}{p_{1}}}, & \text { if } \beta_{2} \leq 0, \tau<0,
\end{array}
\end{aligned}
$$

for $\frac{n}{p^{*}}<\delta=\frac{\beta_{1}}{p_{1}}$ and $\tau \neq \max \left(0, \frac{\beta_{2}}{p_{1}}\right)$. But our method of proof gives us for $\tau \geq \frac{\beta_{2}}{p_{1}}+\frac{1}{q_{2}}-\frac{1}{q_{1}}$ instead of (4.34) the estimate

$\varepsilon_{o} \leq c 2^{-k \gamma} N^{\varkappa_{\theta}}\left(\sum_{j=1}^{N} j^{q_{2}\left(\theta \gamma-1 / q_{1}-\frac{\beta_{2}}{p_{1}}\right)}\right)^{1 / q_{2}} \quad$, where $\varkappa_{\theta}=\min \left(\frac{1}{q_{1}}, \theta \gamma-\frac{\beta_{2}}{p_{1}}\right)$.

This leads to the estimate

$e_{k}\left(\mathrm{id}^{\beta_{1}, \beta_{2}}\right) \leq C k^{-\frac{s_{1}-s_{2}}{n}}(1+\log k)^{\tau-\frac{\beta_{2}}{p_{1}}+\varkappa}, \quad$ if $\frac{\beta_{2}}{p_{1}} \leq \frac{\beta_{2}}{p_{1}}+\frac{1}{q_{2}}-\frac{1}{q_{1}}<\tau$,

where $\varkappa=\min \left(\frac{1}{q_{1}}, \tau+\frac{1}{q_{1}}-\frac{1}{q_{2}}-\frac{\beta_{2}}{p_{1}}\right)$. For $q_{1}=\infty$ this means $\varkappa=0$ such that this situation strongly supports our above conjecture: when $q_{1}=\infty$ and $\tau \neq \max \left(0, \frac{\beta_{2}}{p_{1}}\right)$, then we have (4.35) in the limiting case $\frac{n}{p^{*}}<\delta=\frac{\beta_{1}}{p_{1}}$. This special case may be surprising at first glance, but (4.33) explains some reason: the disturbing term $j^{-q_{2} / q_{1}}$ then disappears in the sum such that we do not have to balance both entries and the argument is much simplified. As already pointed out, we therefore believe that (4.35) describes the desired asymptotic behaviour correctly and the two-sided estimate for $\tau \geq \frac{\beta_{2}}{p_{1}}+\frac{1}{q_{2}}-\frac{1}{q_{1}}$ is only due to our method of proof.

In contrast to this consideration, it is not clear what may happen in the limiting case $\tau=\max \left(0, \frac{\beta_{2}}{p_{1}}\right)$ in (4.10), i.e., when $\frac{n}{p^{*}}<\delta=\frac{\beta_{1}}{p_{1}}$; in the majority of cases we have only two-sided estimates here. But this is not even solved for the simpler situation $\beta_{2}=0$, cf. [31, Thm. 2]. 
Remark 4.6. We return to the special situation studied in [12] and recalled in front of Corollary 3.15. Under the same assumptions as there, in particular $a_{1}>a_{2}$ and (3.75), now with the additional restriction that $a_{1}-a_{2} \neq \frac{1}{p_{1}}-\frac{1}{p_{2}}$, then

$$
e_{k}\left(\operatorname{id}_{F, \log }^{\Omega}\right) \sim k^{-\min \left(a_{1}-a_{2}, \frac{1}{p_{1}}-\frac{1}{p_{2}}\right)}, \quad k \in \mathbb{N} .
$$

Theorem 4.7. Let the parameters satisfy (3.14) and let the weight $w_{(\boldsymbol{\alpha}, \boldsymbol{\beta})} \in \mathcal{A}_{\infty}$ be of type (3.21) with (3.22). We assume that

$$
\begin{gathered}
\text { either } \min \left(\delta, \frac{\beta_{1}}{p_{1}}\right)>\frac{n}{p^{*}}, \quad \beta_{2} \in \mathbb{R}, \\
\text { or } \frac{\beta_{1}}{p_{1}}=\frac{n}{p^{*}}, \quad \frac{\beta_{2}}{p_{1}}>\frac{1}{p^{*}},
\end{gathered}
$$

and

$$
\begin{aligned}
\text { either } \quad \delta>\max \left(\frac{\alpha_{1}}{p_{1}}, \frac{n}{p^{*}}\right), \quad \alpha_{2} \in \mathbb{R}, \\
\quad \text { or } \quad \delta=\frac{\alpha_{1}}{p_{1}}>\frac{n}{p^{*}}, \quad \frac{\alpha_{2}}{p_{1}}>\frac{s_{1}-s_{2}}{n}+\frac{\delta}{n} .
\end{aligned}
$$

(i) If $\frac{n}{p^{*}}<\frac{\beta_{1}}{p_{1}}<\delta$ and $\beta_{2} \in \mathbb{R}$, then

$$
e_{k}\left(A_{p_{1}, q_{1}}^{s_{1}}\left(\mathbb{R}^{n}, w_{(\boldsymbol{\alpha}, \boldsymbol{\beta})}\right) \hookrightarrow A_{p_{2}, q_{2}}^{s_{2}}\left(\mathbb{R}^{n}\right)\right) \sim k^{-\frac{\beta_{1}}{n_{1}}-\frac{1}{p_{1}}+\frac{1}{p_{2}}}(1+\log k)^{-\frac{\beta_{2}}{p_{1}}}, \quad k \in \mathbb{N} .
$$

(ii) If $\frac{n}{p^{*}}<\delta<\frac{\beta_{1}}{p_{1}}$ and $\beta_{2} \in \mathbb{R}$, then

$$
e_{k}\left(A_{p_{1}, q_{1}}^{s_{1}}\left(\mathbb{R}^{n}, w_{(\boldsymbol{\alpha}, \boldsymbol{\beta})}\right) \hookrightarrow A_{p_{2}, q_{2}}^{s_{2}}\left(\mathbb{R}^{n}\right)\right) \sim k^{-\frac{s_{1}-s_{2}}{n}}, \quad k \in \mathbb{N} .
$$

(iii) If $\frac{n}{p^{*}}<\delta=\frac{\beta_{1}}{p_{1}}, \beta_{2} \in \mathbb{R}$, and $\tau=\frac{s_{1}-s_{2}}{n}+\frac{1}{q_{2}}-\frac{1}{q_{1}} \neq \max \left(0, \frac{\beta_{2}}{p_{1}}\right)$, then for $k \in \mathbb{N}$,

$e_{k}\left(B_{p_{1}, q_{1}}^{s_{1}}\left(\mathbb{R}^{n}, w_{(\boldsymbol{\alpha}, \boldsymbol{\beta})}\right) \hookrightarrow B_{p_{2}, q_{2}}^{s_{2}}\left(\mathbb{R}^{n}\right)\right) \sim$

$$
\sim \begin{cases}k^{-\frac{s_{1}-s_{2}}{n},}, & \text { if } \frac{\beta_{2}}{p_{1}}>\max (\tau, 0), \\ k^{-\frac{s_{1}-s_{2}}{n}}(1+\log k)^{\tau-\frac{\beta_{2}}{p_{1}}}, & \text { if } \frac{\beta_{2}}{p_{1}} \leq 0<\tau, \\ & \text { or } 0<\frac{\beta_{2}}{p_{1}}<\tau \leq \frac{\beta_{2}}{p_{1}}+\frac{1}{q_{2}}-\frac{1}{q_{1}}, \\ k^{-\frac{s_{1}-s_{2}}{n}}(1+\log k)^{-\frac{\beta_{2}}{p_{1}}}, & \text { if } \beta_{2} \leq 0, \tau<0 .\end{cases}
$$


(iv) In case of $\delta>\frac{\beta_{1}}{p_{1}}=\frac{n}{p^{*}}, \frac{\beta_{2}}{p_{1}}>\frac{1}{p^{*}}$, then for $p^{*}<\infty$ we obtain for all $k \in \mathbb{N}$,

$e_{k}\left(A_{p_{1}, q_{1}}^{s_{1}}\left(\mathbb{R}^{n}, w_{(\boldsymbol{\alpha}, \boldsymbol{\beta})}\right) \hookrightarrow A_{p_{2}, q_{2}}^{s_{2}}\left(\mathbb{R}^{n}\right)\right) \sim(1+\log k)^{-\frac{\beta_{2}}{p_{1}}+\frac{1}{p^{*}}}$,

whereas for $p^{*}=\infty$, that is, $\beta_{1}=0, \beta_{2}>0$,

$$
\begin{aligned}
e_{k}\left(A_{p_{1}, q_{1}}^{s_{1}}\left(\mathbb{R}^{n}, w_{(\boldsymbol{\alpha}, \boldsymbol{\beta})}\right)\right. & \left.\hookrightarrow A_{p_{2}, q_{2}}^{s_{2}}\left(\mathbb{R}^{n}\right)\right) \sim \\
& \sim \begin{cases}k^{-\frac{\beta_{2}}{p_{1}}}, & \text { if } \frac{\beta_{2}}{p_{1}} \leq \frac{1}{p_{1}}-\frac{1}{p_{2}}, \\
k^{-\frac{1}{p_{1}}+\frac{1}{p_{2}}}(1+\log k)^{-\frac{\beta_{2}}{p_{1}}+\frac{1}{p_{1}}-\frac{1}{p_{2}}}, & \text { if } \frac{\beta_{2}}{p_{1}}>\frac{1}{p_{1}}-\frac{1}{p_{2}} .\end{cases}
\end{aligned}
$$

Proof. We follow the idea of the proof of Theorem 4.1 and can reduce our consideration to the case

$$
e_{k}\left(B_{p_{1}, q_{1}}^{s_{1}}\left(\mathbb{R}^{n}, w\right) \hookrightarrow B_{p_{2}, q_{2}}^{s_{2}}\left(\mathbb{R}^{n}\right)\right) \sim e_{k}\left(\ell_{q_{1}}\left(2^{j \delta} \ell_{p_{1}}\left(\widetilde{w}_{(\boldsymbol{\alpha}, \boldsymbol{\beta})}\right)\right) \hookrightarrow \ell_{q_{2}}\left(\ell_{p_{2}}\right)\right),
$$

where

$$
\widetilde{w}_{(\boldsymbol{\alpha}, \boldsymbol{\beta})}(j, l)= \begin{cases}(1+j)^{\alpha_{2}} & \text { if } l=0 \\ \left(2^{-j} l\right)^{\frac{\alpha_{1}}{n}}\left(1-\log \left(2^{-j} l\right)\right)^{\alpha_{2}} & \text { if } 2^{-j} l<1, \\ \left(2^{-j} l\right)^{\frac{\beta_{1}}{n}}\left(1+\log \left(2^{-j} l\right)\right)^{\beta_{2}} & \text { if } 2^{-j} l \geq 1 .\end{cases}
$$

Clearly, in view of (3.2), we immediately get the $F$-result in cases (i), (ii), and (iv) since no dependence on the $q$-parameters appears.

We divide the identity operator Id : $\ell_{q_{1}}\left(2^{j \delta} \ell_{p_{1}}\left(\widetilde{w}_{(\boldsymbol{\alpha}, \boldsymbol{\beta})}\right)\right) \hookrightarrow \ell_{q_{2}}\left(\ell_{p_{2}}\right)$ into two parts $\mathrm{Id}=\mathrm{Id}_{1}+\mathrm{Id}_{2}$, where

$\operatorname{Id}_{1}: \ell_{q_{1}}\left(2^{j \delta} \ell_{p_{1}}^{j^{j n}}\left(\widetilde{w}_{(\boldsymbol{\alpha}, \boldsymbol{\beta})}\right)\right) \hookrightarrow \ell_{q_{2}}\left(\ell_{p_{2}}\right)$ and $\operatorname{Id}_{2}: \ell_{q_{1}}\left(2^{j \delta} \tilde{\ell}_{p_{1}}^{2^{j n}}\left(\widetilde{w}_{\alpha, \beta}\right)\right) \hookrightarrow \ell_{q_{2}}\left(\ell_{p_{2}}\right)$.

In case of (4.39) we can find numbers $\bar{\alpha}_{1}$ and $\underline{\alpha}_{1}$ such that

$$
\bar{\alpha}_{1}>\alpha_{1}>\underline{\alpha}_{1}>-n \text { and } \max \left(\frac{n}{p^{*}}, \frac{\bar{\alpha}_{1}}{p_{1}}\right)<\delta .
$$

Thus by Corollary 3.11 with $\gamma=\left(\bar{\alpha}_{1}, 0\right), \boldsymbol{\beta}=\varkappa$, we obtain

$$
\begin{aligned}
e_{k}\left(\ell_{q_{1}}\left(2^{j \delta} \ell_{p_{1}}\left(\widetilde{w}_{(\boldsymbol{\alpha}, \boldsymbol{\beta})}\right)\right) \hookrightarrow \ell_{q_{2}}\left(\ell_{p_{2}}\right)\right) & \sim e_{k}\left(B_{p_{1}, q_{1}}^{s_{1}}\left(\mathbb{R}^{n}, w_{(\boldsymbol{\alpha}, \boldsymbol{\beta})}\right) \hookrightarrow B_{p_{2}, q_{2}}^{s_{2}}\left(\mathbb{R}^{n}\right)\right) \\
& \leq c e_{k}\left(B_{p_{1}, q_{1}}^{s_{1}}\left(\mathbb{R}^{n}, w_{(\boldsymbol{\gamma}, \boldsymbol{\beta})}\right) \hookrightarrow B_{p_{2}, q_{2}}^{s_{2}}\left(\mathbb{R}^{n}\right)\right) \\
& \sim e_{k}\left(\ell_{q_{1}}\left(2^{j \delta} \ell_{p_{1}}\left(\widetilde{w}_{(\boldsymbol{\gamma}, \boldsymbol{\beta})}\right)\right) \hookrightarrow \ell_{q_{2}}\left(\ell_{p_{2}}\right)\right),
\end{aligned}
$$


where

$$
\widetilde{w}_{(\boldsymbol{\gamma}, \boldsymbol{\beta})}(j, l)= \begin{cases}1 & \text { if } l=0 \\ \left(2^{-j} l\right)^{\frac{\boldsymbol{\alpha}_{1}}{n}} & \text { if } 2^{-j} l<1 \\ \left(2^{-j} l\right)^{\frac{\beta_{1}}{n}}\left(1+\log \left(2^{-j} l\right)\right)^{\beta_{2}} & \text { if } 2^{-j} l \geq 1\end{cases}
$$

Now it follows from Lemma 4.3 with $\theta=\delta-\frac{\bar{\alpha}_{1}}{p_{1}}$ and $\xi=\frac{\bar{\alpha}_{1}}{n}$ that

$$
e_{k}\left(\operatorname{Id}_{1}\right) \leq c e_{k}\left(\overline{\operatorname{Id}}_{1}\right) \sim k^{-\left(\frac{\delta}{n}+\frac{1}{p_{1}}-\frac{1}{p_{2}}\right)}=k^{-\frac{s_{1}-s_{2}}{n}}
$$

(in obvious notation). On the other hand, interchanging the rôle of $\boldsymbol{\alpha}$ and $\gamma=\left(\underline{\alpha}_{1}, 0\right), \boldsymbol{\varkappa}=\boldsymbol{\beta}$, in Corollary 3.11 we obtain the inequality converse to (4.43),

$$
e_{k}\left(\operatorname{Id}_{1}\right) \geq c e_{k}\left(\underline{\mathrm{Id}}_{1}\right) \sim k^{-\frac{s_{1}-s_{2}}{n}},
$$

i.e., finally,

$$
e_{k}\left(\operatorname{Id}_{1}\right) \sim k^{-\frac{s_{1}-s_{2}}{n}}, \quad k \in \mathbb{N} .
$$

In the limiting situation (4.40) the argument for the lower estimate remains valid, whereas it is no longer possible to find $\bar{\alpha}_{1}$ with (4.42) since $\delta=\frac{\alpha_{1}}{p_{1}}=\max \left(\frac{\alpha_{1}}{p_{1}}, \frac{n}{p^{*}}\right)$ in this situation. Here we use Remark 4.6 together with the splitting argument presented in Step 3 of the proof of Corollary 3.15. Thus

$$
\begin{aligned}
e_{k}\left(\operatorname{Id}_{1}\right) & \leq c e_{k}\left(\operatorname{id}_{\Omega}: F_{p_{1}, q_{1}}^{s_{1}}\left(\Omega, w_{(\boldsymbol{\alpha}, \boldsymbol{\alpha})}\right) \hookrightarrow F_{p_{2}, q_{2}}^{s_{2}}(\Omega)\right) \\
& \leq c^{\prime} e_{k}\left(\operatorname{id}_{F, \log }^{\Omega}: F_{q_{1}}^{s_{1}}\left(L_{r, r}(\log L)_{\theta}\right)(\Omega) \hookrightarrow F_{p_{2}, q_{2}}^{s_{2}}(\Omega)\right) \\
& \leq c^{\prime \prime} k^{-\min \left(\theta, \frac{1}{p_{2}}-\frac{1}{r}\right)} \sim k^{-\min \left(\theta, \frac{s_{1}-s_{2}}{n}\right)} \\
& \leq C k^{-\frac{s_{1}-s_{2}}{n}},
\end{aligned}
$$

if we can choose $\theta$ such that $0<\frac{s_{1}-s_{2}}{n} \leq \theta<\frac{\alpha_{2}}{p_{1}}-\frac{\alpha_{1}}{n p_{1}}=\frac{\alpha_{2}}{p_{1}}-\frac{\delta}{n}$. Here we used the approach in Step 3 of the proof of Corollary 3.15 together with (4.36) and $\delta=\frac{\alpha_{1}}{p_{1}}$ several times.

The estimates of $e_{k}\left(\operatorname{Id}_{2}\right)$ follow from the estimates for the weights $w^{\beta_{1}, \beta_{2}}(x)$, see Proposition 4.4 .

Remark 4.8. Obviously Theorem 4.7 refines and extends Theorem 4.1. Moreover, it is obvious that only 'weak' weights influence the compactness of the embedding measured in terms of its entropy numbers, more precisely, when either $\frac{\beta_{1}}{p_{1}}<\delta$ or $\frac{\beta_{1}}{p_{1}}=\delta$ with $\beta_{2}<0$. In other words, the weight should not 
increase to rapidly for $|x| \rightarrow \infty$. This phenomenon is not only well-known from our previous considerations in [25], but also from parallel observations for so-called 'admissible' weights in [31-33] with forerunners in $[19,20,27]$. In [26] we discussed similar effects in terms of local singularities of the weight.

The most difficult setting is again - as in the above-mentioned related papers - the 'line' $\delta=\frac{\beta_{1}}{p_{1}}$ where we have not yet obtained asymptotically sharp estimates in all admitted cases in (iii), not to speak about the second 'line' $\delta=\frac{\alpha_{1}}{p_{1}}$ occurring in this context, at least when $0<\alpha_{1}<\beta_{1}$. In the figure below we have indicated the different parameter regions according to Theorem 4.7 in this situation.

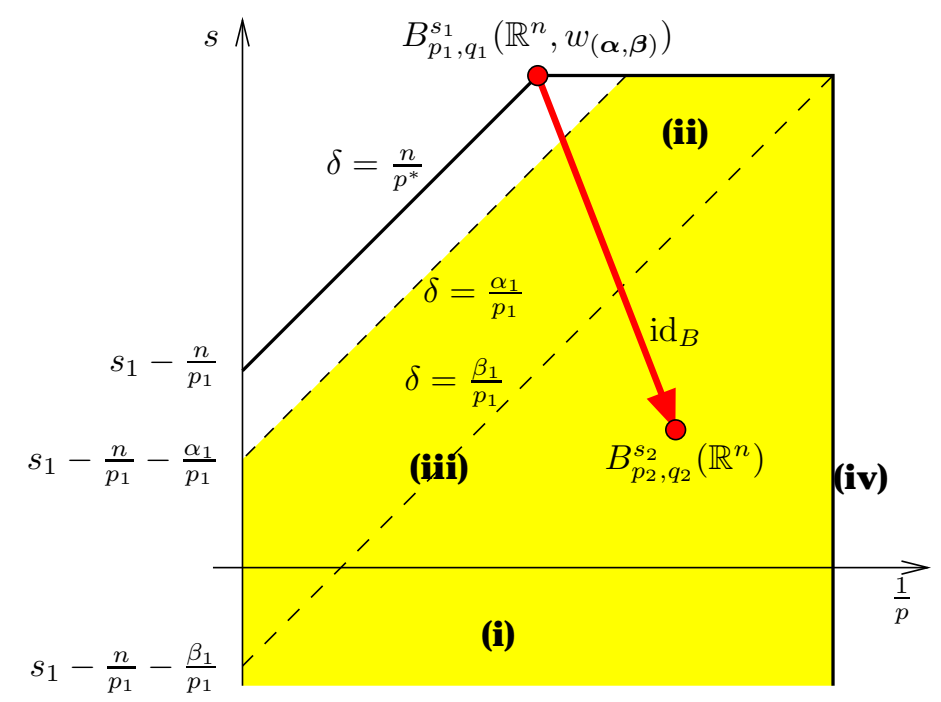

FiguRE 3. The parameter regions according to Theorem 4.7 in case of $0<\alpha_{1}<\beta_{1}$

Remark 4.9. We dealt in [26] with more general weights and proved in [26, Thm. 3.7] the independence of the asymptotic behaviour of related entropy numbers from local singularities represented in our case by the exponents $\boldsymbol{\alpha}=\left(\alpha_{1}, \alpha_{2}\right)$. However, we have to assume in this general setting that $\delta$ is sufficiently large; in our context this leads to $\delta>\frac{n}{p^{*}}+\frac{\max \left(0, \alpha_{1}\right)}{p_{1}}$ which thus excludes the limiting case $\delta=\frac{\alpha_{1}}{p_{1}}$ (even for $p^{*}=\infty$ ), recall Remark 3.17. Studying the unweighted situation on bounded domains, there 
are forerunners of (4.36) for Sobolev spaces of logarithmic type in $[8,12,13]$, in particular, one obtains

$$
e_{k}\left(H_{p_{1}}^{s_{1}}(\log H)_{a_{1}}(\Omega) \rightarrow H_{p_{2}}^{s_{2}}(\log H)_{a_{2}}(\Omega)\right) \sim k^{-\min \left(a_{1}-a_{2}, \frac{s_{1}-s_{2}}{n}\right)}
$$

(neglecting further assumptions for the moment). Not regarding obvious differences one should thus expect some influence of $\alpha_{2}$ in this second limiting case.

Assume that we have $\alpha_{1}=\alpha_{2}=0$ in Theorem 4.7 such that (4.39) can be rewritten as $\delta>\frac{n}{p^{*}}$. Then $w_{(\mathbf{0}, \boldsymbol{\beta})}$ becomes an admissible weight in the sense of the papers [19,20,27,31-33] and Theorem 4.7 reproduces and extends known results on entropy numbers of such embeddings.

Corollary 4.10. Let the parameters satisfy (3.14) and let the weight $w \in \mathcal{A}_{\infty}$ be of type (3.21) with (3.22) and $\alpha_{1}=\alpha_{2}=0$. We assume that

$$
s_{1}-s_{2}>n\left(\frac{1}{p_{1}}-\frac{1}{p_{2}}\right)_{+}
$$

and

$$
\text { either } \min \left(\delta, \frac{\beta_{1}}{p_{1}}\right)>\frac{n}{p^{*}}, \quad \beta_{2} \in \mathbb{R}, \quad \text { or } \quad \frac{\beta_{1}}{p_{1}}=\frac{n}{p^{*}}, \quad \frac{\beta_{2}}{p_{1}}>\frac{1}{p^{*}} .
$$

(i) If $\frac{n}{p^{*}}<\frac{\beta_{1}}{p_{1}}<\delta$ and $\beta_{2} \in \mathbb{R}$, then

$e_{k}\left(A_{p_{1}, q_{1}}^{s_{1}}\left(\mathbb{R}^{n}, w\right) \hookrightarrow A_{p_{2}, q_{2}}^{s_{2}}\left(\mathbb{R}^{n}\right)\right) \sim k^{-\frac{\beta_{1}}{n p_{1}}-\frac{1}{p_{1}}+\frac{1}{p_{2}}}(1+\log k)^{-\frac{\beta_{2}}{p_{1}}}, \quad k \in \mathbb{N}$.

(ii) If $\frac{n}{p^{*}}<\delta<\frac{\beta_{1}}{p_{1}}$ and $\beta_{2} \in \mathbb{R}$, then

$$
e_{k}\left(A_{p_{1}, q_{1}}^{s_{1}}\left(\mathbb{R}^{n}, w\right) \hookrightarrow A_{p_{2}, q_{2}}^{s_{2}}\left(\mathbb{R}^{n}\right)\right) \sim k^{-\frac{s_{1}-s_{2}}{n}}, \quad k \in \mathbb{N} .
$$

(iii) If $\frac{n}{p^{*}}<\delta=\frac{\beta_{1}}{p_{1}}, \beta_{2} \in \mathbb{R}$, and $\tau=\frac{s_{1}-s_{2}}{n}+\frac{1}{q_{2}}-\frac{1}{q_{1}} \neq \max \left(0, \frac{\beta_{2}}{p_{1}}\right)$, then for $k \in \mathbb{N}$, 
$e_{k}\left(B_{p_{1}, q_{1}}^{s_{1}}\left(\mathbb{R}^{n}, w\right) \hookrightarrow B_{p_{2}, q_{2}}^{s_{2}}\left(\mathbb{R}^{n}\right)\right) \sim$

$$
\sim \begin{cases}k^{-\frac{s_{1}-s_{2}}{n}}, & \text { if } \frac{\beta_{2}}{p_{1}}>\max (\tau, 0), \\ k^{-\frac{s_{1}-s_{2}}{n}}(1+\log k)^{\tau-\frac{\beta_{2}}{p_{1}}}, & \text { if } \frac{\beta_{2}}{p_{1}} \leq 0<\tau, \\ & \text { or } 0<\frac{\beta_{2}}{p_{1}}<\tau \leq \frac{\beta_{2}}{p_{1}}+\frac{1}{q_{2}}-\frac{1}{q_{1}}, \\ k^{-\frac{s_{1}-s_{2}}{n}}(1+\log k)^{-\frac{\beta_{2}}{p_{1}}}, & \text { if } \beta_{2} \leq 0, \tau<0 .\end{cases}
$$

(iv) In case of $\delta>\frac{\beta_{1}}{p_{1}}=\frac{n}{p^{*}}, \frac{\beta_{2}}{p_{1}}>\frac{1}{p^{*}}$, then for $p^{*}<\infty$ we obtain for all $k \in \mathbb{N}$,

$$
\begin{gathered}
e_{k}\left(A_{p_{1}, q_{1}}^{s_{1}}\left(\mathbb{R}^{n}, w\right) \hookrightarrow A_{p_{2}, q_{2}}^{s_{2}}\left(\mathbb{R}^{n}\right)\right) \sim(1+\log k)^{-\frac{\beta_{2}}{p_{1}}+\frac{1}{p^{*}}}, \\
\text { whereas for } p^{*}=\infty, \text { that is, } \beta_{1}=0, \beta_{2}>0, \\
e_{k}\left(A_{p_{1}, q_{1}}^{s_{1}}\left(\mathbb{R}^{n}, w\right) \hookrightarrow A_{p_{2}, q_{2}}^{s_{2}}\left(\mathbb{R}^{n}\right)\right) \sim \\
\sim \begin{cases}k^{-\frac{\beta_{2}}{p_{1}}}, & \text { if } \frac{\beta_{2}}{p_{1}} \leq \frac{1}{p_{1}}-\frac{1}{p_{2}}, \\
k^{-\frac{1}{p_{1}}+\frac{1}{p_{2}}}(1+\log k)^{-\frac{\beta_{2}}{p_{1}}+\frac{1}{p_{1}}-\frac{1}{p_{2}}}, & \text { if } \frac{\beta_{2}}{p_{1}}>\frac{1}{p_{1}}-\frac{1}{p_{2}} .\end{cases}
\end{gathered}
$$

Remark 4.11. Cases (i) and (ii) (for $B$-spaces) are covered by [32, Thms. 5.9, 5.13], case (iv) with $p^{*}=\infty$ by [33, Cor. 2] and for $p^{*}<\infty$ by [29, Thm. 7] with some forerunners in [17,20]. Case (iii) with $\beta_{2}=0$ can be found in [31, Thm. 2] and some partial results for $\beta_{2} \neq 0$ in [30, Cor. 7]. The rest is new. Recall that we have for $q_{1}=\infty$ even a complete description in the sense of Remark 4.5. As mentioned above, we believe that (iii) can be extended in all cases (when $\tau \neq \max \left(0, \frac{\beta_{2}}{p_{1}}\right)$ ) to

$$
e_{k}\left(B_{p_{1}, q_{1}}^{s_{1}}\left(\mathbb{R}^{n}, w\right) \hookrightarrow B_{p_{2}, q_{2}}^{s_{2}}\left(\mathbb{R}^{n}\right)\right) \sim k^{-\frac{s_{1}-s_{2}}{n}}(1+\log k)^{\left(\tau_{+}-\frac{\beta_{2}}{p_{1}}\right)_{+}},
$$

but have no proof yet.

Acknowledgement. We are especially indebted to our friend and colleague Th. Kühn who quickly corresponded to a question of us with some new result in [29]. 


\section{References}

[1] C. Bennett and K. Rudnick, On Lorentz-Zygmund spaces, Dissertationes Math., 175 (1980), 72 pp.

[2] M. Bownik, Atomic and molecular decompositions of anisotropic Besov spaces, Math. Z., 250 (2005), 539-571.

[3] M. Bownik and K.P. Ho, Atomic and molecular decompositions of anisotropic Triebel-Lizorkin spaces, Trans. Amer. Math. Soc., 358 (2006), 1469-1510.

[4] H.-Q. Bui, Weighted Besov and Triebel spaces: Interpolation by the real method, Hiroshima Math. J., 12 (1982), 581-605.

[5] H.-Q. Bui, Characterizations of weighted Besov and TriebelLizorkin spaces via temperatures, J. Funct. Anal., 55 (1984), 39-62.

[6] H.-Q. Bui, M. Paluszyński, and M.H. Taibleson, A maximal function characterization of weighted Besov-Lipschitz and Triebel-Lizorkin spaces, Studia Math., 119 (1996), no. 3, 219246.

[7] H.-Q. Bui, M. Paluszyński, and M.H. Taibleson, Characterization of the Besov-Lipschitz and Triebel-Lizorkin spaces. The case $q<1$, J. Fourier Anal. Appl., 3 (1997), Spec. Iss., 837-846.

[8] A. Caetano, Entropy numbers of embeddings between logarithmic Sobolev spaces, Port. Math., 57 (2000), no. 3, 355-379.

[9] B. Carl and I. Stephani, Entropy, compactness and the approximation of operators, Cambridge Univ. Press, Cambridge, 1990.

[10] F. Cobos, L.M. Fernández-Cabrera, A. Manzano, and A. Martínez, Logarithmic interpolation spaces between quasiBanach spaces, Z. Anal. Anwendungen, 26 (2007), 65-86.

[11] D.E. Edmunds and W.D. Evans, Spectral theory and differential operators, Clarendon Press, Oxford, 1987.

[12] D.E. Edmunds and Yu. Netrusov, Entropy numbers of embeddings of Sobolev spaces in Zygmund spaces, Studia Math., 128 (1998), no. 1, 71-102.

[13] D.E. Edmunds and H. Triebel, Function spaces, entropy numbers, differential operators, Cambridge Univ. Press, Cambridge, 1996. 
[14] R. Farwig and H. Sohr, Weighted $L^{q}$-theory for the Stokes resolvent in exterior domains, J. Math. Soc. Japan, 49 (1997), no. 2, 251-288.

[15] M. Frazier and S. Roudenko, Matrix-weighted Besov spaces and conditions of $\mathcal{A}_{p}$ type for $0<p \leq 1$, Indiana Univ. Math. J., 53 (2004), no. 5, 1225-1254.

[16] J. García-Cuerva and J. L. Rubio de Francia, Weighted norm inequalities and related topics, volume 116 of North-Holland Mathematics Studies. North-Holland, Amsterdam, 1985.

[17] D. Haroske, Some limiting embeddings in weighted function spaces and related entropy numbers, Forschungsergebnisse Math/Inf/97/04, Universität Jena, Germany (1997), 54 pp.

[18] D. Haroske, Some logarithmic function spaces, entropy numbers, applications to spectral theory, Dissertationes Math., 373 (1998), 59 pp.

[19] D. Haroske and H. Triebel, Entropy numbers in weighted function spaces and eigenvalue distribution of some degenerate pseudodifferential operators I, Math. Nachr., 167 (1994), 131156.

[20] D.D. Haroske, Embeddings of some weighted function spaces on $\mathbb{R}^{n}$; entropy and approximation numbers. A survey of some recent results, An. Univ. Craiova, Ser. Mat. Inform., vol. XXIV (1997), 1-44.

[21] D.D. Haroske, Logarithmic Sobolev spaces on $\mathbb{R}^{n}$; entropy numbers, and some application, Forum Math., 12 (2000), no. 3, 257-313.

[22] D.D. Haroske, Envelopes and sharp embeddings of function spaces, volume 437 of Chapman \& Hall/CRC Research Notes in Mathematics, Chapman \& Hall/CRC, Boca Raton, FL, 2007.

[23] D.D. Haroske, Sobolev spaces with Muckenhoupt weights, singularities and inequalities, Georgian Math. J., 15 (2008), no. 2, 263-280.

[24] D.D. Haroske and I. Piotrowska, Atomic decompositions of function spaces with Muckenhoupt weights, and some relation to fractal analysis, Math. Nachr., 281 (2008), no. 10, 14761494.

[25] D.D. Haroske and L. Skrzypczak, Entropy and approximation numbers of embeddings of function spaces with Muckenhoupt weights, I, Rev. Mat. Complut., 21 (2008), no. 1, 135-177. 
[26] D.D. Haroske and L. Skrzypczak, Entropy and approximation numbers of embeddings of function spaces with Muckenhoupt weights, II. General weights, Ann. Acad. Sci. Fenn. Math., 36 (2011), 111-138.

[27] D.D. Haroske and H. Triebel, Wavelet bases and entropy numbers in weighted function spaces, Math. Nachr., 278 (2005), no. 1-2, 108-132.

[28] Th. Kühn, Entropy numbers of general diagonal operators, Rev. Mat. Complut., 18 (2005), no. 2, 479-491.

[29] Th. Kühn, Entropy numbers in sequence spaces with an application to weighted function spaces, J. Approx. Theory, 153 (2008), no. 1, 40-52.

[30] Th. Kühn, H.-G. Leopold, W. Sickel, and L. Skrzypczak, Entropy numbers of embeddings of weighted Besov spaces, Jenaer Schriften zur Mathematik und Informatik Math/Inf/13/03, Universität Jena, Germany, (2003), 57 pp.

[31] Th. Kühn, H.-G. Leopold, W. Sickel, and L. Skrzypczak, Entropy numbers of embeddings of weighted Besov spaces, Constr. Approx., 23 (2006), 61-77.

[32] Th. Kühn, H.-G. Leopold, W. Sickel, and L. Skrzypczak, Entropy numbers of embeddings of weighted Besov spaces II, Proc. Edinburgh Math. Soc. (2), 49 (2006), 331-359.

[33] Th. Kühn, H.-G. Leopold, W. Sickel, and L. Skrzypczak, Entropy numbers of embeddings of weighted Besov spaces III. Weights of logarithmic type, Math. Z., 255 (2007), no. 1, 1-15.

[34] C. Merucci, Applications of interpolation with a function parameter to Lorentz, Sobolev and Besov spaces, In M. Cwikel and J. Peetre, editors, Interpolation spaces and allied topics in analysis, volume 1070 of LNM, pages 183-201. Proc. Conf., Lund/Swed. 1983, Springer, 1984.

[35] B. Muckenhoupt, Hardy's inequality with weights, Studia Math., 44 (1972), 31-38.

[36] B. Muckenhoupt, Weighted norm inequalities for the Hardy maximal function, Trans. Amer. Math. Soc., 165 (1972), 207226.

[37] B. Muckenhoupt, The equivalence of two conditions for weight functions, Studia Math., 49 (1973/74), 101-106.

[38] A. Pietsch, Eigenvalues and s-numbers, Akad. Verlagsgesellschaft Geest \& Portig, Leipzig, 1987. 
[39] S. Roudenko, Matrix-weighted Besov spaces, Trans. Amer. Math. Soc., 355 (2002), 273-314.

[40] S. Roudenko, Duality of matrix-weighted Besov spaces, Studia Math., 160 (2004), no. 2, 129-156.

[41] V.S. Rychkov, Littlewood-Paley theory and function spaces with $A_{p}^{\text {loc }}$ weights, Math. Nachr., 224 (2001), 145-180.

[42] W. Sickel and H. Triebel, Hölder inequalities and sharp embeddings in function spaces of $B_{p, q}^{s}$ and $F_{p, q}^{s}$ type, $Z$. Anal. Anwendungen, 14 (1995), 105-140.

[43] E.M. Stein, Harmonic analysis, real-variable methods, orthogonality, and oscillatory integrals, Princeton Univ. Press, Princeton, 1993.

[44] J.-O. Strömberg and A. Torchinsky, Weighted Hardy spaces, volume 1381 of LNM, Springer, Berlin, 1989.

[45] A. Torchinsky, Real-variable methods in harmonic analysis, volume 123 of Pure and Applied Mathematics, Academic Press Inc., Orlando, FL, 1986.

[46] H. Triebel, Theory of function spaces, Birkhäuser, Basel, 1983.

[47] H. Triebel, Theory of function spaces II, Birkhäuser, Basel, 1992.

[48] H. Triebel, Fractals and spectra, Birkhäuser, Basel, 1997.

[49] H. Triebel, Theory of function spaces III, Birkhäuser, Basel, 2006.

Mathematical Institute

Friedrich-Schiller-University Jena

D-07737 Jena, Germany

(E-mail : dorothee.haroske@uni-jena.de)

Faculty of Mathematics \& Computer Science

Adam Mickiewicz University, Ul. Umultowska 87

61-614 Poznań, Poland

(E-mail : lskrzyp@amu.edu.pl)

(Received : July 2009) 


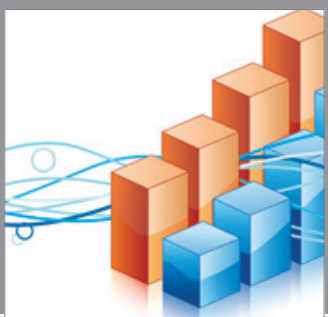

Advances in

Operations Research

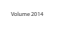

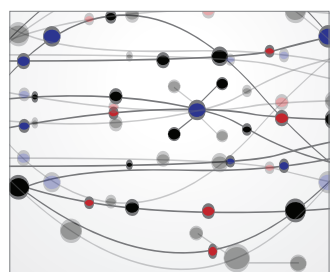

\section{The Scientific} World Journal
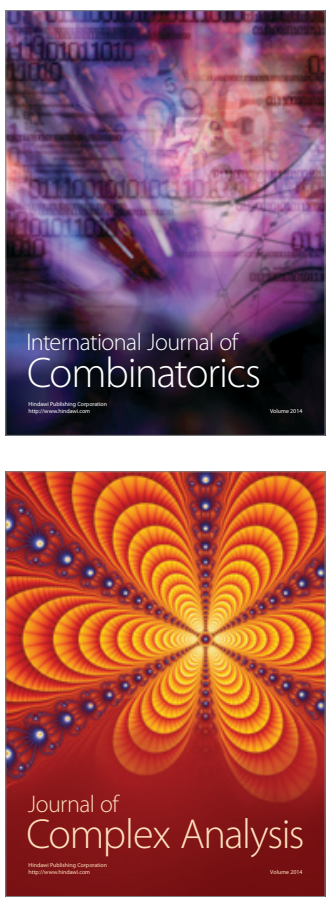

International Journal of

Mathematics and

Mathematical

Sciences
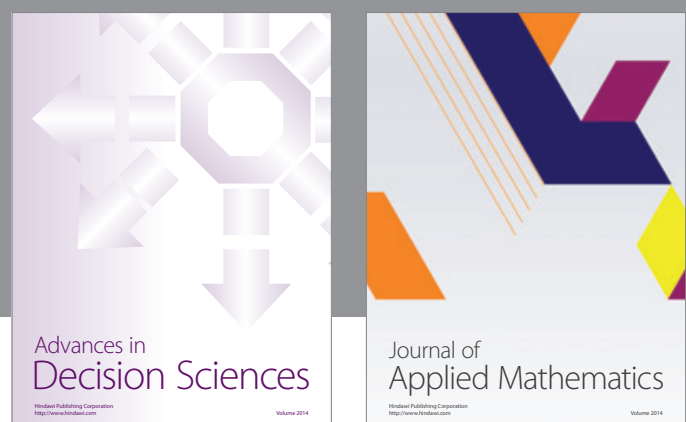

Journal of

Applied Mathematics
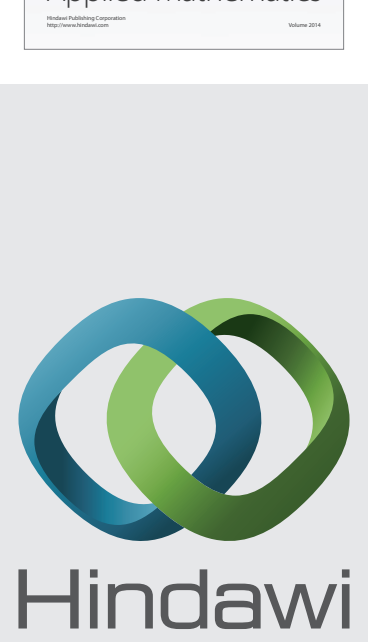

Submit your manuscripts at http://www.hindawi.com
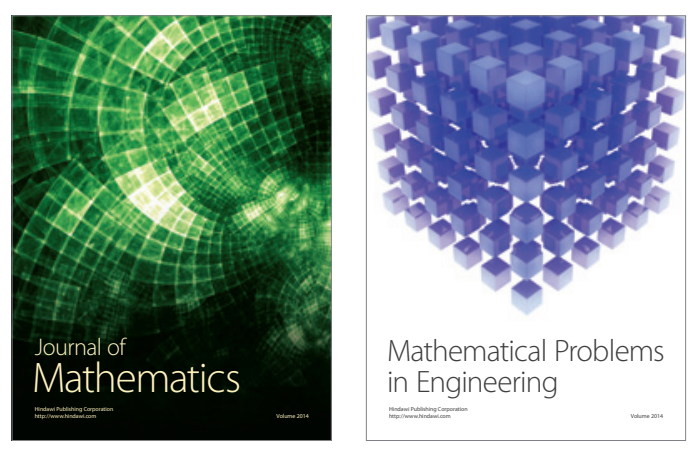

Mathematical Problems in Engineering
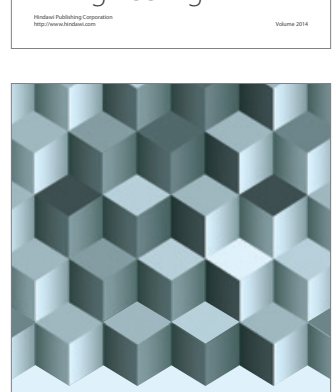

Journal of

Function Spaces
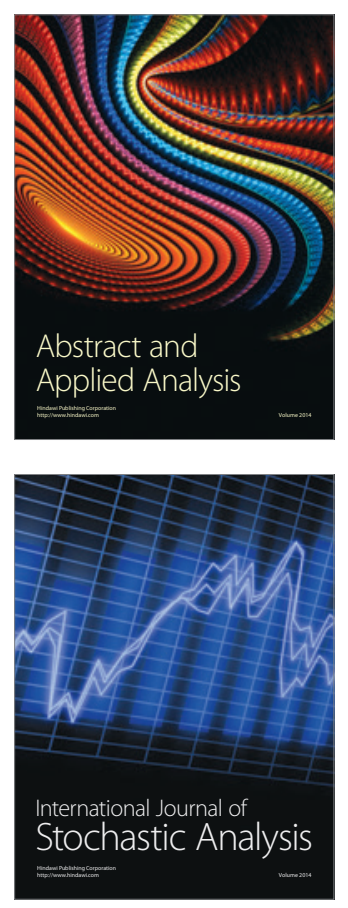

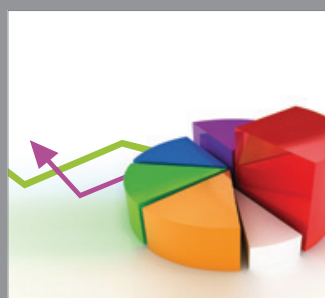

ournal of

Probability and Statistics

Promensencen
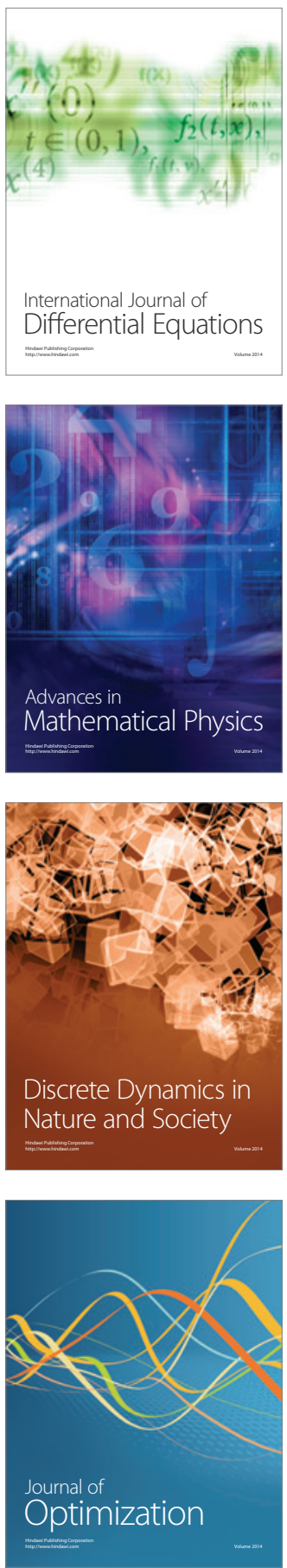